\title{
Nonlinear impact monitoring: Line Of Variation searches for impactors
}

\author{
Andrea Milani ${ }^{a}$, Steven R. Chesley ${ }^{\mathrm{b}}$, \\ Maria Eugenia Sansaturio ${ }^{c}$, Giacomo Tommei ${ }^{\text {a }}$ \\ Giovanni B. Valsecchi ${ }^{\mathrm{d}}$, \\ ${ }^{a}$ Dipartimento di Matematica, Università di Pisa Via Buonarroti 256127 Pisa, \\ Italy \\ b Jet Propulsion Laboratory, CalTech Pasadena, CA 91109, USA \\ ${ }^{\mathrm{c}}$ E.T.S. de Ingenieros Industriales, University of Valladolid Paseo del Cauce $s / n$ \\ 47011 Valladolid, Spain \\ ${ }^{\mathrm{d}}$ IASF-INAF Via Fosso del Cavaliere 10000133 Roma, Italy
}

\begin{abstract}
When a new Near Earth Asteroid is discovered, it is important to know whether or not there is the possibility of an impact with the Earth in the near future. In this paper, we describe the technical approaches employed by the two operational second-generation asteroid impact monitoring systems, CLOMON2 and Sentry, paying particular attention to the similarities and differences between these independent systems. The detection and characterization of a potential impact requires the propagation of the orbital probability density function from the time of discovery to the time of hypothetical impact. Since the N-body problem is not integrable, this can be done only by sampling the orbital elements space with a finite number of Virtual Asteroids (VAs), the orbit of each one being propagated numerically. Our methods, illustrated in this paper, use the Line Of Variation (LOV), a unidimensional subspace, to perform this sampling. The primary goal is to detect Virtual Impactors (VIs), which are regions in the initial conditions space leading to dynamically distinct collision solutions; then a probability integral needs to be computed on the volume of the VI. An important issue is how to assure completeness of such a search down to some impact probability threshold. This problem cannot be efficiently solved just by computing more VAs, but requires
\end{abstract}

Email addresses: milani@dm.unipi.it (Andrea Milani), steve.chesley@jpl.nasa.gov (Steven R. Chesley), genny@pisces.eis.uva.es (Maria Eugenia Sansaturio), tommei@mail.dm.unipi.it (Giacomo Tommei), giovanni@rm.iasf.cnr.it (Giovanni B. Valsecchi). 
a geometric description of the behavior of the LOV in order to identify the critical segments of this curve. We have studied these behaviors on the Target Plane (TP) through our analytical theory and the output of many numerical tests. Assuming that the geometry is the simplest compatible with the data available from the sampling, we obtain a classification which allows us to use iterative methods, appropriate for each case, to find the closest approach distance possible along the LOV. After an LOV minimum has been identified, it is possible to use a probability density linearized at this point. However, when the cross section of the Earth is not crossed by the LOV, there is no guarantee that nonlinearity would be negligible in the direction on the TP transversal to the LOV. We describe how to test for such nonlinearity, and thus reduce or eliminate the possibility of spurious VIs. In this way, the performance of our impact monitoring systems has been significantly increased in comparison to the earlier and simpler solitary system. These more advanced systems have identified and then eliminated (through additional observations) more than one hundred cases of asteroids with VIs in the years 2002-2003.

Key Words: Celestial Mechanics; Asteroids, Dynamics; Impacts 


\section{INTRODUCTION}

As recently as 1998, when the $1997 \mathrm{XF}_{11}$ case arose, efficient algorithms to decide whether a given Near Earth Asteroid (NEA) could impact the Earth in the near future (e.g., in the next 100 years) were not available (Marsden 1999). The Monte Carlo methods could in principle be used, but their computational efficiency is limited by the inverse relationship between probability of impact and the number of Virtual Asteroids (VAs) to be computed (Milani et al. 2002).

In 1999 a new class of algorithms was introduced to scan the confidence region of possible orbits of a newly discovered NEA in order to detect Virtual Impactors (VIs) (Milani et al. 1999). The basic idea was that the set of VAs used to sample the confidence region should not be considered as a set of independent points (in the orbital elements space), but as a geometric object, namely a string, which in turn is sampled by uniformly spaced points. As a result, interpolation between two consecutive VAs along the string is meaningful and this allows us to prove the existence of a closest approach around a given encounter date. This method was computationally efficient to the point that it was possible to detect a VI with a probability of the order of $10^{-9}$ for the asteroid $1999 \mathrm{AN}_{10}$.

The next advance was to introduce an iterative algorithm (a variant of Newton's method) to find the minimum possible approach distance in a neighborhood of a given VA having a close encounter with the Earth (Milani et al. 2000a). This enabled the development of an automatic monitoring system, CLOMON (Chesley and Milani 2000), that processed all newly discovered NEAs in order to detect VIs in the next 50 (later 80) years. For each NEA with low Minimum Orbital Intersection Distance (MOID) the software robot CLOMON computed 1,200 alternate orbital solutions, uniformly sampling the string covering a segment of the Line Of Variation (LOV) around the nominal solution (Milani 1999). Then the orbit of each VA was propagated for the search time span and the list of all close approaches was stored. This list was scanned looking for cases in which there were VAs not only having deep encounters, but such that by a small change in the initial conditions a much closer approach was possible. Newton's method was started from each VA satisfying these conditions; given the minimum distance achieved at convergence of the iterations, if the confidence region computed at convergence included a portion of the Earth cross section, a record was written in a risk file with an admittedly rough estimate of the impact probability.

The CLOMON system has been operational since November 1999 and the output risk files have been available on the web. The main purpose of this system was to alert the observers of the need to follow up the NEAs for 
which impacts are possible, so that such possibility could be contradicted by new observations. This has indeed happened in dozens of cases (successful observational campaigns to remove VIs are documented on the Spaceguard Central Node site ${ }^{1}$ ).

Given the purpose of the CLOMON robot as an alert system, its fundamental requirement was to be complete, not in the sense of detecting all the VIs for some asteroid, but in the sense of finding all the asteroids having some VIs. According to our current understanding of the limitations of CLOMON, its weak points were the following.

- CLOMON was the only operational system for about two years, and during this period there was no redundant checking and no possibility of crossverification of results from an independent system.

- The computation of the multiple solutions to be used as VAs was based on variables that are not always well adapted when the observed arc was very short.

- The choice of the asteroids for which the scan had to be performed did not consider all of the relevant parameters, and so some asteroids that harbored VIs were neglected.

- The VAs were propagated without the variational equations, and thus the uncertainty at each encounter was unknown until a subsequent in-depth analysis with Newton's method. Unfortunately, it was generally unclear whether such an analysis was even necessary until it was completed, and worse, in many cases where it was necessary it was never even conducted.

- The method of Newton did not guarantee convergence, indeed it was divergent in a very large number of cases, in which no conclusion about the existence of a specific VI could be reached.

- The extrapolation of results obtained along the LOV in the transversal direction on the target plane could lead to spurious VIs.

The performance of CLOMON over its first two years of operation was satisfactory, but there was substantial room for improvement, as well as an obvious need for an independent and redundant system operating in parallel. This paper describes the two second-generation systems, both of which became operational in early 2002, that have taken the place of CLOMON, the lone first-generation system. CLOMON2 ${ }^{2}$, operating at the University of Pisa and the University of Valladolid, represents a major overhaul of the precursor CLOMON system, while Sentry ${ }^{3}$, operated at the Jet Propulsion Laboratory (JPL), was developed as part of a new initiative. Although the two systems are substantially independent in the sense that they do not share any software

\footnotetext{
1 http://spaceguard.esa.int/SSystem/NEOCS/Successful.html

2 http://newton.dm.unipi.it/neodys/, see the Risk Page.

3 http://neo.jpl.nasa.gov/risk/
} 
and many of the analysis techniques are different, their joint development has been the result of intensive discussions and they do share a number of fundamental concepts. In this article we describe the two systems, highlighting the most important differences between them and emphasizing the common advances over the first-generation CLOMON.

This paper is organized as follows.

- Section 2 provides a general treatment of problems investigated and solutions adopted by the new systems.

- Section 3 describes an analytical approach that helps us to understand the mechanisms responsible for encounters.

- Sections 4, 5, 6 and 7 illustrate the algorithms used by Sentry and CLOMON2 to solve the problems introduced in Sec. 2.

- Section 8 presents some conclusions and discusses possible future work to further improve our systems.

\section{IMPACT MONITORING OVERVIEW}

\subsection{COMPUTATION OF THE MULTIPLE SOLUTIONS}

The first step in the search for VIs is the LOV sampling. The LOV is a differentiable curve in the orbital elements space, parameterized by a real parameter $\sigma$ related to the RMS of the observation residuals. There are effective algorithms to sample the LOV, that is to compute multiple solutions (Milani, 1999; Milani et al., 2004).

Multiple solutions sampling is coordinate system dependent. However, when the observed arc is long enough, the confidence region is so much elongated that its sampling by the LOV is representative, with whatever coordinates. If on the contrary the observed arc is short (1-2 days), then the confidence region is wide in two directions and any 1-dimensional LOV cannot sample it in a reliable way. Different LOVs, computed with different coordinates, provide independent sampling and may provide different results (Milani et al., 2004, Figures 1-4)

CLOMON2 routinely uses equinoctial elements, but also runs in cartesian coordinates. Sentry routinely uses cometary elements but can optionally run in cartesian or keplerian. It is important for redundancy that the different coordinate systems are used. As an example, CLOMON2 has accumulated a long list of cases where the search for VIs has been performed both in equinoctal and in cartesian coordinates. The results have been consistently better, with 
more VIs detected, with the LOV computed in cartesian coordinates whenever the asteroid had been observed only over an arc spanning less than 2 degrees on the celestial sphere. For much longer arcs the orbit determination is more linear in orbital elements (better equinoctal for low $e$, cometary for high $e$ ).

Once an LOV coordinate system has been selected, the LOV is sampled with a prescribed density over a specified range of values for the parameter $\sigma$. CLOMON2 samples the LOV with either 1,201 or 2,401 uniformly-spaced VAs in the interval $\sigma \in[-3,3]$. Sentry samples 10,001 multiple solutions over $\sigma \in[-5,5]$ with a stepsize according to a modified Gaussian probability density at the local LOV $\sigma$ value.

\subsection{SCHEDULING OF COMPUTATIONS}

Given the rate at which new NEAs are discovered and reobserved, and the computing power available to us, we cannot scan all objects for VIs at every orbital update. Thus, with a simple computation, we need to carefully limit the set of asteroids for which a complete computation must be performed. This is done by selecting and prioritizing the objects based on two criteria; they must be able to pass near the Earth and they should possess enough orbital uncertainty so that purely linear approaches may not reveal all VIs. (For highly refined orbits, linear methods are sufficient to reveal VIs.) Thus we define a score as a function of the MOID and the RUNOFF, which is the along track uncertainty obtained from an exploratory 100-year forward integration. The RUNOFF is proportional to the uncertainty in the encounter timing.

The CLOMON equation for base score was

$$
S=50-25 \cdot\left[80 \cdot \mathrm{MOID}-\log _{10}(\mathrm{RUNOFF})\right]
$$

where both MOID and RUNOFF are in AU (Chesley and Milani 2000). The MOID was computed at the nominal solution at the time of the observations.

When the search for VIs has already been done once, we multiply $S$ by a factor taking into account the change since the time of the previous computation, in such a way that cases with only slight differences from the previous search receive a lower score and cases just scanned have a zero score. A priority list is formed, sorted by the value of the score, and the VI scan always starts from the top of the list.

The main problem with the CLOMON scoring system was that the MOID is highly uncertain whenever the asteroid is discovered far from the Earth. Note that this happens for the largest asteroids, precisely those for which even a minute impact probability has to be rated relevant. In these cases the MOID 
computed from the nominal least squares solution could be large - indeed the asteroid may not even be rated as a Potentially Hazardous Asteroid because its MOID exceeds $0.05 \mathrm{AU}$ - and still initial conditions with MOID $=0$ could be well within the confidence region.

In the second generation systems, although the overall logic is the same, the base score is computed in a better way. We compute multiple solutions for each NEA with large RUNOFF (in excess of 0.7 AU for CLOMON2 and 1.0 AU for Sentry) in order to have information on the uncertainty of the MOID. We use in the score formula above the minimum value of the MOID among the multiple solutions, in place of the nominal MOID. (Sentry uses the minimum of the multiple solutions MOIDs and the minimum MOID obtained during a 100-year orbit propagation). Thus, if the LOV crosses the MOID = 0 line, the score is practically dependent only upon the RUNOFF, with $S>0$ for RUNOFF $>0.01 \mathrm{AU}$.

As an example, the asteroid $1997 \mathrm{AE}_{12}$ had been observed over an arc of only 6 days in 1997; at the time it was at 1.48 AU from the Earth. It was never scanned by CLOMON, because the nominal MOID was 0.09 AU. With the introduction of MOID uncertainty, $1997 \mathrm{AE}_{12}$ was queued for analysis, and promptly several VIs were found, although with very small impact probability. (1997 $\mathrm{AE}_{12}$ was serendipitously re-discovered in 2003, resulting in the elimination of all VIs.)

\subsection{TARGET PLANES}

Each VA obtained from the multiple solutions method is integrated forward to some point in the future (until 2080 for CLOMON2 and for 100 years for Sentry), logging all close approach information obtained during the propagation.

CLOMON did not propagate the variational equations nor save uncertainty information for the individual VAs, thus it had no uncertainty information about the encounter. This lack of uncertainty information proved to be a serious handicap. In contrast, the second-generation systems described here map the linearized uncertainty for each VA to the time of each encounter. This allows an early and quick assessment of whether a potential encounter could be threatening, and enables a clearer interpretation of the tracks formed by the LOV on the target plane, as described in Sec. 4.

Before describing further how impact monitoring works, let us introduce the target plane, which plays a fundamental role in the study of planetary encounters. As described in Milani et al. 2002, there are two ways to define such a plane: the Target Plane (TP), or b-plane, used in Öpik theory, and the Mod- 
ified Target Plane (MTP), introduced by Milani and Valsecchi 1999. Both of them contain the center of the Earth; the TP is normal to the incoming asymptote of the geocentric hyperbola on which the small body travels when it is closest to the planet, while the MTP is normal to the geocentric velocity vector of the small body at closest approach.

While CLOMON used only the MTP, CLOMON2 and Sentry perform their target plane analyses in the TP by default. All data are still stored in the MTP reference frame, but then the systems transform the MTP data to the $\mathrm{TP}$ reference frame, except in unusual cases where the encounter is too slow (if there is temporary satellite capture of the asteroid the TP would not be defined). The reason for switching to the TP is that nearby trajectories are deflected by different amounts; this has practically no effect on the orientation of the TP, which is related to the pre-encounter velocity vector of the asteroid. On the other hand, the MTPs of nearby trajectories are oriented differently, because of the deflection of the velocity vector in the vicinity of the Earth; if this deflection is significant, it leads to a strong nonlinearity in the mapping from the asteroid's pre-encounter state onto the TP. After many encounters of the asteroid the nonlinearity would become too strong and our algorithms would become less efficient.

The details of the transformation from the MTP to the TP are given in Appendix A. Hereafter we shall use the acronym TP to indicate the target plane in use, that is the $b$-plane, if it is defined, otherwise (or when we are speaking of CLOMON) the Modified Target Plane.

\subsection{TARGET PLANE GEOMETRY AND ALGORITHMS}

The original CLOMON algorithms were based upon the geometric idea of propagating the motion of a string of possible orbital solutions. However, once a suitable close approach of one of the VAs was found, the one dimensional strategy was abandoned in favor of a two dimensional search on the TP of the encounter under consideration. This search extended outside of the image of the string on the TP, so that the nonlinearity of the orbit propagation might easily result in divergence of such unconstrained iterations.

A key idea of the new systems was to define algorithms to search for a minimum possible encounter distance without leaving the string, that is the LOV. Thus the task can be described as the minimization of a function of one variable $r^{2}$, the closest approach distance (around a given date) squared, as a function of the parameter $\sigma$ along the LOV. The function $r^{2}(\sigma)$ is differentiable, thus we can search for zeroes of the derivative $f(\sigma)=d\left(r^{2}\right) / d \sigma$. Guaranteed convergence can be obtained under the following conditions: if there is a closed 
interval $\left[\sigma_{1}, \sigma_{2}\right]$ such that a close approach occurs (to the same planet, and around the same date) for all initial conditions corresponding to a parameter $\sigma \in\left[\sigma_{1}, \sigma_{2}\right]$, and the values of the derivative at the two extremes are $f\left(\sigma_{1}\right)<0$ and $f\left(\sigma_{2}\right)>0$, then there is at least one minimum of $r^{2}(\sigma)$ inside the interval. Moreover, under these hypotheses, the regula falsi algorithm provides an iterative procedure converging to some stationary point (almost always a minimum).

This approach has obvious advantages as far as reliability is concerned, with a computational cost comparable to the method used in CLOMON. There are two drawbacks. First, to start the regula falsi procedure we need at least two consecutive VAs having encounters around the same date, thus tracing two sequential points on some TP. Second, the minimum possible distance along the LOV could be more than one Earth radius and still an impact could be possible, because the image on the $\mathrm{TP}$ of the entire confidence region is not just a line but has some width.

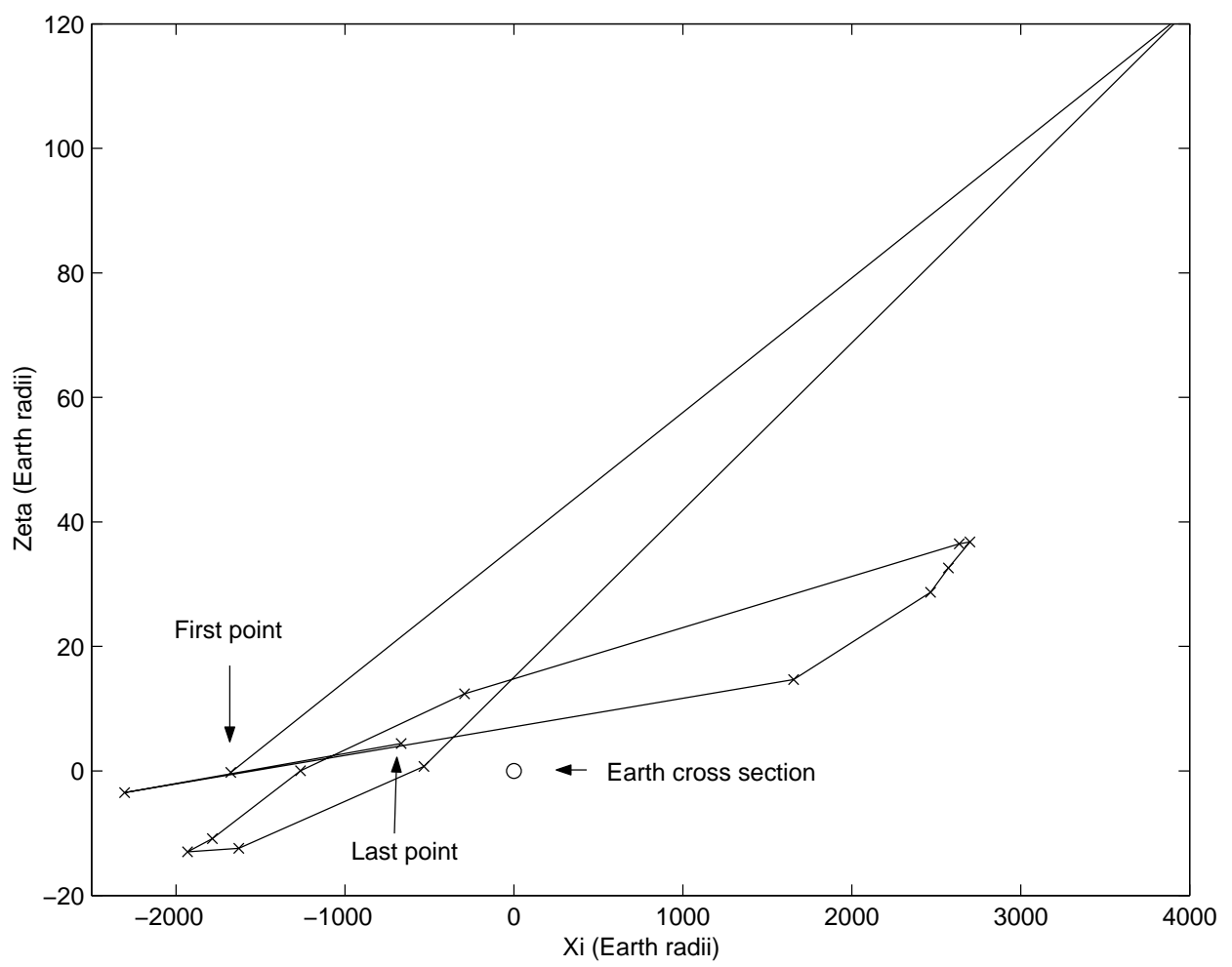

Fig. 1. A trail of the asteroid $2003 \mathrm{UM}_{3}$ on the TP of the Earth in 2040. By using only the information contained in the first and last point it would be impossible to deduce the behavior of the LOV segment in between, because the geometry is very far from being the simplest possible. The cross-section of the Earth is not to scale.

If there is only one TP point then we have a singleton, and the only solution is to resort to a unidimensional Newton's method. CLOMON2 implements a variant which searches for zeroes of $f(\sigma)$ with controlled length steps, in such a way that divergence is not possible, provided that the image on the TP exists 
for some neighborhood of the first LOV point. Nevertheless this method can fail because the iterations may lead to a point on the LOV which misses the TP. In practice this happens, because an isolated TP point indicates a very nonlinear situation. To increase the number of cases in which at least two TP points are available, CLOMON2 has increased the radius of the disk on the TP to $0.2 \mathrm{AU}$ (CLOMON used 0.1 AU). As we shall see in the next section, Sentry is able to ignore singletons and still detect low probability VIs because of its denser sampling of the LOV.

These changes have resulted in a sharp decrease in the relative number of divergent cases. However, even starting with two TP points the procedure cannot be always convergent. Our algorithms are based on the principle of the simplest geometry, by which we model the qualitative behavior of the LOV image on the TP in the simplest possible way, compatible with the available information on the TP points. This behavior, as discussed in Section 3, becomes more complex as a result of each subsequent close approach. Therefore there is no absolute limit on the complexity of the figure drawn by the LOV on a TP: Figure 1 shows a typical example of chaotic behavior. As in fractal sets, smaller and smaller subintervals on the LOV can be distorted according to a typical pattern. The principle of the simplest geometry allows our algorithms to terminate within acceptable computation times by not following finer and finer details.

\subsection{GENERIC LEVEL OF COMPLETION OF THE VI SCAN}

How complete is the search for VIs? As we shall see, this is a difficult question to answer rigorously, but it can be useful to understand how efficient a system could be under idealized circumstances. Specifically, we can ask what is the highest impact probability VI that could possibly escape detection if the associated trail on the TP is fully linear. This we call the generic completion level of the system.

Under these generic assumptions, the VI stream will simply be a narrow and straight trail passing through the center of the TP; a diametrical chord. If a sufficient number of VAs (one for CLOMON2, two for Sentry) intersect the target plane then the methods described in the previous subsection will reveal the VI. Otherwise, the VI will be missed in the scan.

The generic completion level is given by

$$
\mathrm{IP}^{*}=\frac{\delta p}{\Delta \mathrm{TP}}
$$

where $\delta p$ is the integral probability between TP points and $\Delta \mathrm{TP}$ is the maximal separation between TP points, measured in Earth diameters $\left(D_{\oplus}\right)$, for 
which a VI detection is assured.

For CLOMON2, the TP radius is $0.2 \mathrm{AU}$, and, under our generic hypothesis, only one point on the TP is required to detect a VI. Thus the spacing of consecutive VAs on the target plane can be no more than $0.4 \mathrm{AU} \simeq 4700 D_{\oplus}$. CLOMON2 samples 1200 VAs over the interval $|\sigma| \leq 3$ on the LOV, and so these points are separated by $\Delta \sigma=0.005$, and the maximum cumulative probability over one interval is $\delta p=\Delta \sigma / \sqrt{2 \pi} \simeq 0.002$. From this we can compute the CLOMON2 generic completeness,

$$
\mathrm{IP}_{\mathrm{CLOMON} 2}^{*} \simeq 0.002 / 4700 \simeq 4.3 \times 10^{-7}
$$

In contrast, Sentry requires two VAs to intersect the TP within $0.1 \mathrm{AU}$ of the Earth, and so the maximum separation for guaranteed VI detection (under our baseline assumptions) is $0.1 \mathrm{AU} \simeq 1175 D_{\oplus}$, a factor 4 smaller than CLOMON2. Sentry samples VAs in 10,000 uniform steps in integral probability, covering $|\sigma| \leq 5$, and so to good accuracy $\delta p=10^{-4}$, which is a factor 20 smaller than the corresponding CLOMON2 number. Thus, we obtain the Sentry generic completeness,

$$
\mathrm{IP}_{\text {Sentry }}^{*} \simeq 10^{-4} / 1175 \simeq 8.6 \times 10^{-8}
$$

The Sentry propagation expense is a factor of $10000 / 1200 \simeq 8$ times higher than CLOMON2, which yields a factor 5 increase in generic completion. Thus CLOMON2 is somewhat more efficient than Sentry, due in large part to the analysis of singletons on the TP. This highlights a key philosophical difference between the two systems, which is that CLOMON2 propagates fewer VAs, conducting a more exhaustive search for VIs with the output. This approach necessitates careful attention to TP nonlinearities. In contrast, Sentry strives to minimize the detrimental effects of TP nonlinearities by applying a much denser sampling of the LOV, which will assure multiple TP points of streams that possess VIs with significant impact probabilities.

As a result of the choices of the parameters, and of the efforts at optimization, both systems have an acceptably prompt response to the arrival of new NEA astrometry. Average CPU times per object are in the range of 1.5-2 hours per object on an Athlon MP1900+-class processor (although rare cases can require 5-6 hours). Thus it is possible to scan about a dozen newly discovered and/or re-observed asteroids per day (per processor), enough to cope with even the busiest days. 


\subsection{ELIMINATION OF SPURIOUS VIs}

After computing the minimum close approach distance along the LOV we need to establish whether an impact that is compatible with the available observations is possible. If the TP point obtained in the minimization procedure corresponds to a collision then there must be a small connected region in the confidence region (in the initial conditions space) leading to impact at the given date. This region is what we call a VI, and the problem is how to compute an Impact Probability (IP) associated with it.

However, the TP point of minimum distance along the LOV could be outside the impact cross section and nevertheless a VI could exist. This happens because the image of the confidence region is not just the LOV, but more like a strip around it. The basic approach is to linearize the map between the initial conditions space and the TP. Then the confidence ellipsoid in the initial conditions space is mapped onto an elliptic disk on the TP. If this disk intersects the impact cross section there is a VI.

To compute an IP we can use the Gaussian formalism. If the probability distribution of the initial conditions is described by a normal distribution (in 6-dimensional space) with the boundary ellipsoids of the confidence regions as level surfaces, then the probability distribution of the TP points is described by a normal distribution (in 2 dimensions) with the above mentioned ellipses as level curves. The coefficients of the normal distribution on the TP can be explicitly computed (see, e.g., Milani and Valsecchi 1999) and then it is possible to compute the probability integral of this distribution on the impact cross section (Milani et al. 2002). Of course the normal distributions are never exactly zero, thus formally the IP would be always positive even for very distant close approaches; however, when the probability formally computed in this way is minute, e.g., $<10^{-11}$ (CLOMON2) or $<10^{-10}$ (Sentry) we just dismiss it ${ }^{4}$.

This approach gives a reasonable approximation of the IP under the following assumptions.

(1) The multiple solutions sample well the confidence region in the orbital elements space.

(2) The distance on the TP between the Earth and the LOV point B of closest

\footnotetext{
4 This approach is consistent with what Gauss himself suggested in the original work (Gauss 1809): [the error function] would appropriately be referred to the class of discontinuous [non-analytic] functions, and if we undertake to substitute any analytical function in the place of it for practical purposes, this must be of such a form that it may converge to zero on both sides, asymptotically, ..., so that beyond this limit it can be regarded as actually vanishing.
} 
approach is small, allowing for the linearization in B of the Gaussian probability density to represent well the probability density at the Earth.

The fact is that both assumptions can fail. When a NEA has recently been discovered, and has been observed for only a few days, the confidence region is very large. In particular, when an asteroid is discovered far from the Earth, the value of the MOID is highly uncertain (Bonanno 2000). This uncertainty is reflected in a large semi-width, the minor axis of the TP confidence ellipse. Then the probability density at the impact points, estimated with the normal distribution, can be significant even at large distances from the TP point on the LOV, and conversely the probability distribution, if computed exactly accounting for the nonlinearity, could be unexpectedly low at points very close to the LOV.

The failure of the linearized Gaussian approximation can result in spurious VIs, that is cases in which the approximated IP is non-negligible, while an exact probability computation would give an "actually vanishing" result. Note that for our purposes it is neither essential to find all the VIs of a given asteroid, nor to avoid all the spurious ones. The essential requirement is to have a list of VIs which is not empty when, and only when, the real list of VIs with non-vanishing probability is not empty. In this way the asteroid can be marked as "to be reobserved," and the later observations will allow recomputation. However, it is possible to end up with a computed list of VIs consisting only of spurious ones. Even in this case, to request additional observations is reasonable, but it would be better to have an algorithm to filter out the spurious VIs, to the extent that this can be done reliably. Both CLOMON2 and Sentry have implemented different methods of filtering spurious cases from the list of VIs.

The "spurious VI elimination" algorithm of CLOMON2 is based on the principle that to prove the existence of a VI one must explicitly exhibit an initial condition (the representative of the VI) such that

(1) it leads to a collision;

(2) the residuals of the available observations are acceptable (Muinonen 1999).

Thus, starting from the TP point corresponding to the minimum approach distance along the LOV, if there appears to be a VI in the linear approximation, CLOMON2 applies a Newton method (similar to the one used by CLOMON) to obtain an initial condition leading to a TP point inside the impact cross section. If this iterative procedure fails (as described in Section 7) the VI is rated as spurious. If the procedure converges to an explicit VI, the IP is rescaled by using the RMS of the observation residuals; in practice, if the collision orbit results in unacceptable residuals, the VI is also considered spurious. The problem with this approach is that Newton's method does not 
have guaranteed convergence, thus it may be impossible to confirm a VI which is nonetheless real.

Sentry takes a significantly different approach in eliminating spurious VIs, and that is to test for excessive nonlinearity at the LOV's point of closest approach to the Earth. If the local behavior is highly nonlinear, as judged by the curvature of the LOV and the rate of change of stretching along the LOV, then the VI detection is presumed unreliable and discarded. This approach has the opposite problem with respect to that of CLOMON2, in the sense that it does allow some spurious VIs to pass unfiltered. Nonetheless, this is judged a preferred alternative to the possibility of inadvertently eliminating a significant VI. Even so, the risk of incorrectly rejecting a real VI is present in both systems' approaches, but such cases can be expected to have fairly slight impact probabilities due to the extreme nonlinearity that is present.

\section{PROPAGATION OF THE LOV}

In the vast majority of cases, the sequence of VAs along the LOV on the TP of a planetary encounter is spaced along track, i.e., in mean anomaly. This spacing is the consequence of a corresponding spacing in semimajor axis that, in turn, is due to the uncertainty in the initial semimajor axis, for the first encounter along the integration, and also to the amplification of the initial dispersion in semimajor axis, for the subsequent encounters. Given a disk around the Earth, on the TP, the LOV image in most cases crosses the disk, but there are cases in which the LOV enters and goes out from the same side with respect to the Earth (interrupted return, see Milani et al 2000b and Valsecchi et al 2003). In general, the tips (reversal points) of the interrupted returns correspond to local maxima or minima of the semimajor axis. In the following we use the analytic theory of Valsecchi et al. 2003 to find expressions for the local maxima and minima of the semimajor axis that have been induced by a planetary encounter, and discuss the behavior of the stretching at these points. The notation is the same used in that paper, to which we refer the reader for the details of the theory; we limit ourselves hereafter to the definitions.

The theory is an extension of that by Öpik 1976, in which the small body moves on a heliocentric unperturbed orbit until it comes in the vicinity of a planet moving on a circular heliocentric orbit. Here, "in the vicinity" means within a suitably defined "sphere of action", in which the gravity of the planet dominates over that of the Sun; for instance, we can use "Hill's sphere", that for the Earth has radius equal to the distance to the Lagrangian points $L_{1}$ and $L_{2}$ of the Sun-Earth-small body system, that is $0.01 \mathrm{AU}$.

When the small body is in the vicinity of the planet, the motion is assumed 
to be planetocentric, hyperbolic, and not perturbed by the Sun; moreover, the effect of the planet is considered as an instantaneous rotation of the planetocentric velocity vector $\vec{U}$, oriented along the incoming asymptote of the planetocentric hyperbola, to the vector $\vec{U}^{\prime}$, of the same magnitude, oriented along the outgoing asymptote of the same hyperbola. The transition takes place at the moment in which the small body crosses the TP, and allows us to define a post-encounter TP that contains the planet and is orthogonal to $\overrightarrow{U^{\prime}}$.

This approximation allows us to compute the effects of a close planetary encounter with explicit analytical expressions, but is valid only as long as

- the planetocentric orbit is hyperbolic;

- the time in which the rotation of the velocity vector takes place along the real trajectory of the small body is negligible compared to the heliocentric orbital period.

The vector $\vec{U}$ is conveniently described by its magnitude $U$ and two angles, $\theta$ and $\phi$. In a planetocentric reference frame in which the Sun is on the negative $X$-axis, and the $Y$-axis is directed along the motion of the planet, these angles are defined as follows: $\theta$ is the angle from the $Y$-axis to $\vec{U}$, and $\phi$ is the angle, counted clockwise, from the plane $Y-Z$ to the plane $Y-\vec{U}$. It is customary in Öpik's theory to express the magnitude of $\vec{U}$ in units of the orbital velocity of the planet ${ }^{5}$, with the further simplification of neglecting terms of the order of the miss distance. In this approximation the values of $U, \theta$, and $\phi$ are simple functions of the elements $a, e, i$ of the pre-encounter heliocentric orbit of the small body.

The vector $\vec{b}$, defined on the TP, joins the center of the planet to the point in which $\vec{U}$ crosses the TP; the components of $\vec{b}$ on the TP are $\xi$ and $\zeta$, with the latter oriented in the direction opposite to the projection of the $Y$-axis (the direction of motion of the planet) on the TP. The local MOID is $|\xi|$, and differences in $\zeta$ turn out to be differences only in the timing of the encounter. The magnitude $b$ of $\vec{b}$ is the "impact parameter", and the TP is also called the $b$-plane.

The rotation from $\vec{U}$ to $\overrightarrow{U^{\prime}}$ takes place about the straight line passing through the planet and normal to $\vec{b}$; the rotation angle $\gamma$ is given by:

$$
\tan \frac{\gamma}{2}=\frac{m}{b U^{2}}=\frac{c}{b}
$$

Here, $m$ is the mass of the planet in units of that of the Sun, and we have introduced $c=m / U^{2}$, a quantity conserved at successive close encounters

$\overline{5}$ Disregarding the contribution of the mass of the planet in computing its velocity. 
with the same planet ${ }^{6}$, that has the dimension of a length; if the small body passes at $b=c$, then $\gamma=\pi / 2$.

In the rest of this Section we will make use of the same convention as in Valsecchi et al. 2003, denoting by a prime the quantities referred to the postencounter TP, and with a double prime those referred to the TP of the next encounter.

\subsection{THE WIRE APPROXIMATION}

The "wire approximation" used in Valsecchi et al. 2003, Section 5, consists in the assumption of a continuous stream of particles, all with the same $U$, $\theta, \phi$ and $\xi$, and differing only for $\zeta$ (and $t_{0}$, an ignorable coordinate, that we will continue to ignore hereafter ${ }^{7}$. The $\zeta$ coordinate on the TP is linearly related to the timing of the encounter. The LOV image on the TP is mostly spread along the $\zeta$ direction, because of the difference in timing of encounter due to the spread in mean motion. Thus the wire approximation is useful, although it does not consider the spread in the pre-encounter mean motion (see Subsection 3.2).

Local minima and maxima of the post-encounter semimajor axis in the wire approximation

We want to compute, in the simplest possible way, the position of the local minima and maxima of the semimajor axis after a planetary encounter. Since the post-first-encounter semimajor axis $a^{\prime}$ is monotonically related to the postfirst-encounter value of the angle $\theta^{\prime}$,

$$
\begin{aligned}
a^{\prime} & =\frac{1}{1-U^{2}-2 U \cos \theta^{\prime}} \\
\frac{\partial a^{\prime}}{\partial \zeta} & =\frac{2 U}{\left(1-U^{2}-2 U \cos \theta^{\prime}\right)^{2}} \frac{\partial \cos \theta^{\prime}}{\partial \zeta}
\end{aligned}
$$

the problem of finding the local maxima and minima of $a^{\prime}$ as functions of $\zeta$ reduces to that of finding the zeroes of $\partial \cos \theta^{\prime} / \partial \zeta$.

${ }^{6}$ In the approximation where the eccentricity of the planet is $0, U$ is a function of the Jacobi integral apart from terms containing the mass of the planet.

7 There is a connection between our approximation and Gauss' averaging (Murray and Dermott, 2000), which is performed over the same wire. However, in this work we try to handle precisely the cases in which Gauss' averaging is not a good approximation because the close approach is in the neigbourhood of a singularity (Gronchi and Milani, 1999). 
In the wire approximation, with $\xi=\xi_{0}, \cos \theta^{\prime}$ is given by

$$
\cos \theta^{\prime}=\frac{\left(\xi_{0}^{2}+\zeta^{2}-c^{2}\right) \cos \theta+2 c \zeta \sin \theta}{\xi_{0}^{2}+\zeta^{2}+c^{2}}
$$

The derivative of $\cos \theta^{\prime}$ with respect to $\zeta$ is

$$
\frac{\partial \cos \theta^{\prime}}{\partial \zeta}=\frac{2 c\left[\left(\xi_{0}^{2}+c^{2}\right) \sin \theta+2 c \zeta \cos \theta-\zeta^{2} \sin \theta\right]}{\left(\xi_{0}^{2}+\zeta^{2}+c^{2}\right)^{2}}
$$

since the denominator is always positive, the problem is to find the zeroes of the numerator. The latter is of degree 2 in $\zeta$, so there are only a point of maximum $\left(\zeta_{+}\right)$and a point of minimum $\left(\zeta_{-}\right)$of $\cos \theta^{\prime}$ (thus of $a^{\prime}$ ) to be found:

$$
\begin{aligned}
0 & =\zeta^{2}-2 c \zeta \frac{\cos \theta}{\sin \theta}-\left(\xi_{0}^{2}+c^{2}\right) \\
\zeta_{ \pm} & =\frac{c \cos \theta}{\sin \theta} \pm \sqrt{\frac{c^{2}}{\sin ^{2} \theta}+\xi_{0}^{2}} \\
\zeta_{+}-\zeta_{-} & =2 \sqrt{\frac{c^{2}}{\sin ^{2} \theta}+\xi_{0}^{2}} .
\end{aligned}
$$

The difference $\zeta_{+}-\zeta_{-}$gives us the size of the most interesting region, where the largest variations of final semimajor axis take place.

We can also compute the coordinates on the post-first-encounter TP, $\xi_{ \pm}^{\prime}$ and $\zeta_{ \pm}^{\prime}$, corresponding to $\zeta_{ \pm}$:

$$
\begin{aligned}
\xi_{ \pm}^{\prime} & =\frac{\left(\xi_{0}^{2}+\zeta_{ \pm}^{2}+c^{2}\right) \xi_{0} \sin \theta}{\sqrt{\left[\left(\xi_{0}^{2}+\zeta_{ \pm}^{2}-c^{2}\right) \sin \theta-2 c \zeta_{ \pm} \cos \theta\right]^{2}+4 c^{2} \xi_{0}^{2}}} \\
\zeta_{ \pm}^{\prime} & =\frac{\left(\xi_{0}^{2}+\zeta_{ \pm}^{2}-c^{2}\right) \zeta_{ \pm} \sin \theta-2\left(\xi_{0}^{2}+\zeta_{ \pm}^{2}\right) c \cos \theta}{\sqrt{\left[\left(\xi_{0}^{2}+\zeta_{ \pm}^{2}-c^{2}\right) \sin \theta-2 c \zeta_{ \pm} \cos \theta\right]^{2}+4 c^{2} \xi_{0}^{2}}}
\end{aligned}
$$

Adding a keplerian propagation, as in Valsecchi et al. 2003, we can then find the TP coordinates of the second encounter $\xi^{\prime \prime}$ and $\zeta^{\prime \prime}$. In fact, if the heliocentric motion between encounters is keplerian along the orbit characterized by $a^{\prime}, e^{\prime}, i^{\prime}$, then it can be shown that the TP of the second encounter, defined by $\theta^{\prime \prime}, \phi^{\prime \prime}$, is oriented like the post-first-encounter TP: the keplerian propagation does not change the elements of the orbit, so that $\theta^{\prime \prime}=\theta^{\prime}, \phi^{\prime \prime}=\phi^{\prime}$.

For $\xi_{0}^{2}>>c^{2} / \sin \theta$ (an approximation that holds well for the 2028 and the 2027 encounters with the Earth of, respectively, $1997 \mathrm{XF}_{11}$ and $1999 \mathrm{AN}_{10}$ ) the various expressions become simpler: 


$$
\begin{aligned}
\cos \theta^{\prime} & \approx \frac{\left(\xi_{0}^{2}+\zeta^{2}\right) \cos \theta+2 c \zeta \sin \theta}{\xi_{0}^{2}+\zeta^{2}} \\
\frac{\partial \cos \theta^{\prime}}{\partial \zeta} & \approx \frac{2 c\left[\left(\xi_{0}^{2}-\zeta^{2}\right) \sin \theta+2 c \zeta \cos \theta\right]}{\left(\xi_{0}^{2}+\zeta^{2}\right)^{2}} \\
0 & =\zeta^{2}-2 c \zeta \frac{\cos \theta}{\sin \theta}-\xi_{0}^{2} \\
\zeta_{ \pm} & =\frac{c \cos \theta}{\sin \theta} \pm \xi_{0} \\
\zeta_{+}-\zeta_{-} & =2 \xi_{0} \\
\xi_{ \pm}^{\prime} & \approx \xi_{0} \pm \frac{c \cos \theta}{\sin \theta} \\
\xi_{+}^{\prime}-\xi_{-}^{\prime} & \approx \frac{2 c \cos \theta}{\sin \theta} \\
\zeta_{ \pm}^{\prime} & \approx \pm \xi_{0} \\
\zeta_{+}^{\prime}-\zeta_{-}^{\prime} & \approx 2 \xi_{0} .
\end{aligned}
$$

\subsection{THE MODIFIED WIRE APPROXIMATION}

The idea behind the wire approximation is that of simulating the behavior of a real "trail" that has dispersed in time of encounter with the Earth due to the slightly different semimajor axes of individual particles. This latter feature is missing in the original wire approximation outlined by Valsecchi et al. (2003), and we introduce it here.

We therefore introduce the "modified wire approximation", consisting of a continuous stream of particles, all with the same $U, \phi$ and $\xi$, differing for $\zeta$ and $\theta$ in the following way: the extrema of the wire have coordinates $\left(\zeta_{1}, \theta_{1}\right)$ and $\left(\zeta_{2}, \theta_{2}\right)$, with $\zeta_{1}<\zeta_{2}$ and $\theta_{1}>\theta_{2}$; the generic point on the wire has coordinates $(\zeta, \theta)$, such that

$$
\begin{aligned}
\theta & =\theta_{1}+\left(\zeta-\zeta_{1}\right) \Theta \\
\cos \theta & =\cos \theta_{1} \cos \left[\left(\zeta-\zeta_{1}\right) \Theta\right]-\sin \theta_{1} \sin \left[\left(\zeta-\zeta_{1}\right) \Theta\right] \\
& \approx \cos \theta_{1}-\left(\zeta-\zeta_{1}\right) \Theta \sin \theta_{1} \\
\sin \theta & =\sin \theta_{1} \cos \left[\left(\zeta-\zeta_{1}\right) \Theta\right]+\cos \theta_{1} \sin \left[\left(\zeta-\zeta_{1}\right) \Theta\right] \\
& \approx \sin \theta_{1}+\left(\zeta-\zeta_{1}\right) \Theta \cos \theta_{1},
\end{aligned}
$$

where we have put $\Theta=\left(\theta_{2}-\theta_{1}\right) /\left(\zeta_{2}-\zeta_{1}\right)$, and have taken into account that $\left|\left(\zeta-\zeta_{1}\right) \Theta\right| \ll 1$. As just said, the differences in encounter timing, and thus in $\zeta$, are due to differences in the semimajor axis, and thus in $\theta$, of the individual particles, and because of this we should in general have $\Theta<0$. However, this can be expected only if the first encounter that we examine takes place well after the initial epoch, so that the effects of the differences in semimajor axis 
dominate.

Local minima and maxima of the post-encounter semimajor axis in the modified wire approximation

In the modified wire approximation $\cos \theta^{\prime}$ is given by

$$
\cos \theta^{\prime}=\frac{\left(\xi_{0}^{2}+\zeta^{2}-c^{2}\right)\left[\cos \theta_{1}-\left(\zeta-\zeta_{1}\right) \Theta \sin \theta_{1}\right]+2 c \zeta\left[\sin \theta_{1}+\left(\zeta-\zeta_{1}\right) \Theta \cos \theta_{1}\right]}{\xi_{0}^{2}+\zeta^{2}+c^{2}} .
$$

The derivative of $\cos \theta^{\prime}$ with respect to $\zeta$ is

$$
\begin{aligned}
\frac{\partial \cos \theta^{\prime}}{\partial \zeta}= & \frac{1}{\left(\xi_{0}^{2}+\zeta^{2}+c^{2}\right)^{2}} \cdot\left\{\left[\left(c^{4}-\xi_{0}^{4}\right) \Theta+2 c\left(\xi_{0}^{2}+c^{2}\right)\right] \sin \theta_{1}\right. \\
& -2 c\left(\xi_{0}^{2}+c^{2}\right) \zeta_{1} \Theta \cos \theta_{1}+4 c \zeta\left\{c \zeta_{1} \Theta \sin \theta_{1}+\left[\left(\xi_{0}^{2}+c^{2}\right) \Theta+c\right] \cos \theta_{1}\right\} \\
& \left.-2 \zeta^{2}\left\{\left[\left(\xi_{0}^{2}+2 c^{2}\right) \Theta+c\right] \sin \theta_{1}-c \zeta_{1} \Theta \cos \theta_{1}\right\}-\zeta^{4} \Theta \sin \theta_{1}\right\} .
\end{aligned}
$$

Zeroing the numerator, we obtain a fourth degree equation, that has up to four real roots, and that can be easily solved numerically.

This result is the basis to understand Figure 2 in Milani et al. 2000b showing the post 2027 encounter LOV associated with $1999 \mathrm{AN}_{10}$ in the $a$-e plane. In that plane, the LOV assumes a shape that is qualitatively an $\Omega$; in fact, to obtain the shape of the LOV in the $a-e$ plane from an $\Omega$ it is necessary to twist one of the two short segments at the base towards the inside of the $\Omega$. The four local extrema of $a$ correspond to the four "turning points" that one encounters in drawing an $\Omega$ starting from, say, the lower left corner.

The four turning points can be divided in two pairs that have different origins.

One pair, corresponding to the one found also in the wire approximation, is due to the fact that for a given minimum approach distance, equal or greater than the MOID, there is a finite range of post-encounter semimajor axes accessible, and within this range there must be an absolute maximum and an absolute minimum.

The second pair, that is a new feature of the modified wire approximation, has a completely different origin, that can be understood considering that the VAs in the LOV having larger initial semimajor axis are in delay with respect to the Earth, and so are in the positive $\zeta$ half-plane; these VAs are going to be accelerated by the encounter with the Earth (being in delay, they pass behind the planet and receive a positive pull). For the VAs with large enough $\zeta$, the increase in $a$ is negligible; going closer to the planet, i.e. towards smaller values of $\zeta$, the initial semimajor axis decreases, but the positive variation of 
semimajor axis induced by the encounter increases, so that at a certain point the two variations cancel out. An analogous reasoning applies on the other side of the $\xi$ axis.

As already said, the four roots $\zeta_{1,2,3,4}$ of the equation, obtained zeroing the numerator of the derivative, can be found numerically; the corresponding values of $\xi^{\prime}$ can be used to compute the spacing between the "trails", those of $\zeta^{\prime}$ to compute the initial distance between the tips.

The problem can be somewhat simplified in the case in which $\xi_{0}^{2}>>c^{2}$; in this case

$$
\cos \theta^{\prime} \approx \cos \theta_{1}-\left(\zeta-\zeta_{1}\right) \Theta \sin \theta_{1}+\frac{2 c \zeta\left[\sin \theta_{1}+\left(\zeta-\zeta_{1}\right) \Theta \cos \theta_{1}\right]}{\xi_{0}^{2}+\zeta^{2}}
$$

and

$$
\begin{aligned}
\frac{\partial \cos \theta^{\prime}}{\partial \zeta} \approx & \frac{1}{\left(\xi_{0}^{2}+\zeta^{2}\right)^{2}} \cdot\left\{\xi_{0}^{2}\left(2 c-\xi_{0}^{2} \Theta\right) \sin \theta_{1}-2 c \xi_{0}^{2} \zeta_{1} \Theta \cos \theta_{1}+4 c \xi_{0}^{2} \zeta \Theta \cos \theta_{1}\right. \\
& \left.-2 \zeta^{2}\left[\left(\xi_{0}^{2} \Theta+c\right) \sin \theta_{1}-c \zeta_{1} \Theta \cos \theta_{1}\right]-\zeta^{4} \Theta \sin \theta_{1}\right\}
\end{aligned}
$$

The zeroes of the numerator are easily found for the particular case $\theta_{1}=90^{\circ}$ (a further approximation: in fact, for $1997 \mathrm{XF}_{11}, \theta \approx 84^{\circ}$, and for $1999 \mathrm{AN}_{10}$, $\left.\theta \approx 105^{\circ}\right)$ :

$$
\begin{aligned}
0 & =\zeta^{4}+2 \zeta^{2}\left(\frac{c}{\Theta}+\xi_{0}^{2}\right)-\xi_{0}^{2}\left(\frac{2 c}{\Theta}-\xi_{0}^{2}\right) \\
\zeta_{ \pm}^{2} & =-\frac{c}{\Theta}-\xi_{0}^{2} \pm \frac{c}{\Theta} \sqrt{1+\frac{4 \xi_{0}^{2} \Theta}{c}} \\
& \approx-\frac{c}{\Theta}-\xi_{0}^{2} \pm \frac{c}{\Theta}\left(1+\frac{2 \xi_{0}^{2} \Theta}{c}\right) \\
\zeta_{+}^{2} & \approx \xi_{0}^{2} \\
\zeta_{1,2} & \approx \pm \xi_{0} \\
\zeta_{-}^{2} & \approx-\frac{2 c}{\Theta}-3 \xi_{0}^{2} \\
\zeta_{3,4} & \approx \pm \sqrt{-\frac{2 c}{\Theta}-3 \xi_{0}^{2}} .
\end{aligned}
$$

The first two roots have practically not moved from the locations computed with the wire approximation, perhaps not surprisingly, while the two remaining roots are further away from the planet and, as expected, their location is also a function of $\Theta$. 
Examples: for $1997 \mathrm{XF}_{11}, \xi_{0} \approx 1.9 \cdot 10^{-4}, c \approx 1.4 \cdot 10^{-6}$ and we have ${ }^{8} \Theta=-8.4$. $10^{-4}$, thus $\zeta_{3,4} \approx \pm 0.058$, that is the two outer stationary points correspond to shallow encounters. For $1999 \mathrm{AN}_{10}, \xi_{0} \approx 2.5 \cdot 10^{-4}, c \approx 3.9 \cdot 10^{-6}$ and we have ${ }^{9} \Theta=-2.8 \cdot 10^{-4}$, thus $\zeta_{3,4} \approx \pm 0.17$, which corresponds to a very shallow encounter, in a region where the approximations used in the analytic theory are not appropriate. As these examples show, the stationary points $\zeta_{3,4}$ are more important, in determining the way in which the wire folds, when the gradient of semimajor axis is larger, that is when the observed arc is shorter.

\section{The overall scenario}

The picture that emerges is that the region closest to the planet, where the maximum variation of semimajor axis takes place, has an initial size on the LOV of about $2 \xi_{0}$ ( $\xi_{0}$ is the MOID); this central LOV segment is deformed into a Z-shape with two reversals or tips, each of which can give origin to an interrupted return at a future encounter, and is characterized by a stretching that grows going from the tips to $\zeta=0$.

Beyond the tips at $\zeta \approx \pm \xi_{0}$ follow two more LOV segments in which both the variation of semimajor axis and the stretching decrease; the outer boundaries of these trails are a function not only of $\xi_{0}$, but also of $c$ and $\Theta$, in the simple case we have just computed, and of $\theta$ in the general case. These boundaries also give origin to "tips of interrupted returns" at future encounters and, since the stretching is much lower than for the other type of tips, they are much more important from the hazard point of view.

In the framework of the analytic theory it is possible to explicitly compute the increase of the stretching as a result of the first close encounter. At the points $\zeta_{1,2}$ the increase of the stretching is associated with a decrease of the semiwidth such that the overall linearized map from the first to the second TP is approximately area-preserving. This result was first discovered numerically, but can be proven analytically (see Appendix B). The same property does not hold at the points $\zeta_{3,4}$, where the increase of the stretching is not as large. As a result, the product (stretching $\times$ semi-width) in a sequence of encounters is not conserved but nevertheless does not undergo large changes. Moving farther in either direction beyond these outermost tips $\zeta_{3,4}$ the LOV becomes essentially unchanged by the encounter.

$\overline{8}$ By using only the observations between 6 Dec. 1997 and 11 Mar. 1998.

9 By using only the observations between 13 Jan. 1999 and 20 Apr. 1999. 


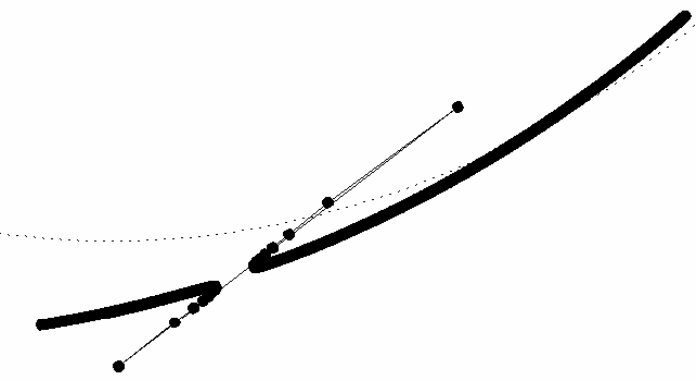

Fig. 2. The VAs of the asteroid $1998 \mathrm{OX}_{4}$ shown as they would be in the year 2002 . The typical $\mathrm{Z}$ shape is the result of the perturbations introduced by an encounter with the Earth in 2001. This asteroid was in fact re-discovered in 2002, thus only one of these VAs is compatible with the present knowledge.

\subsection{FOLDS AND CUSPS}

A return to a close approach, after a previous encounter (after the time of the initial conditions), is mathematically represented as a map between the TP of the first encounter and the TP of the second one. The behavior of such a mapping has been studied by Whitney, 1955 . He proved that generically ${ }^{10}$ the points of the first plane which are singular (that is, at which the differential of the map is not invertible) form smooth curves on the first plane and belong to only two geometrically distinct types: the folds and the cusps.

A fold is such that the image on the second plane of the curve of singular points is a locally regular curve. A cusp of the two-dimensional map corresponds to a cusp (reversal of the tangent vector) on the image on the second TP of the curve of the singular points.

The fold points are such that the mapping can be locally transformed, by suitable differentiable coordinate changes (in both planes), into the normal form

$$
\zeta^{\prime \prime}=\zeta^{2} \quad ; \quad \xi^{\prime \prime}=\xi
$$

where $(\zeta, \xi)$ are the coordinates on the first TP, $\left(\zeta^{\prime \prime}, \xi^{\prime \prime}\right)$ on the second one. In an intuitive way, the first TP folds along the $\zeta=0$ fold line and covers twice the $\zeta^{\prime \prime} \geq 0$ half plane. For the purpose of searching for VIs it is therefore essential to know whether the fold is in the direction from the fold line towards the Earth rather than away from it.

Since this is a generic behavior, it can be easily found in both the analytical

$\overline{{ }^{10} \text { For a }}$ set of mappings dense in the function space of all the differentiable mappings. 

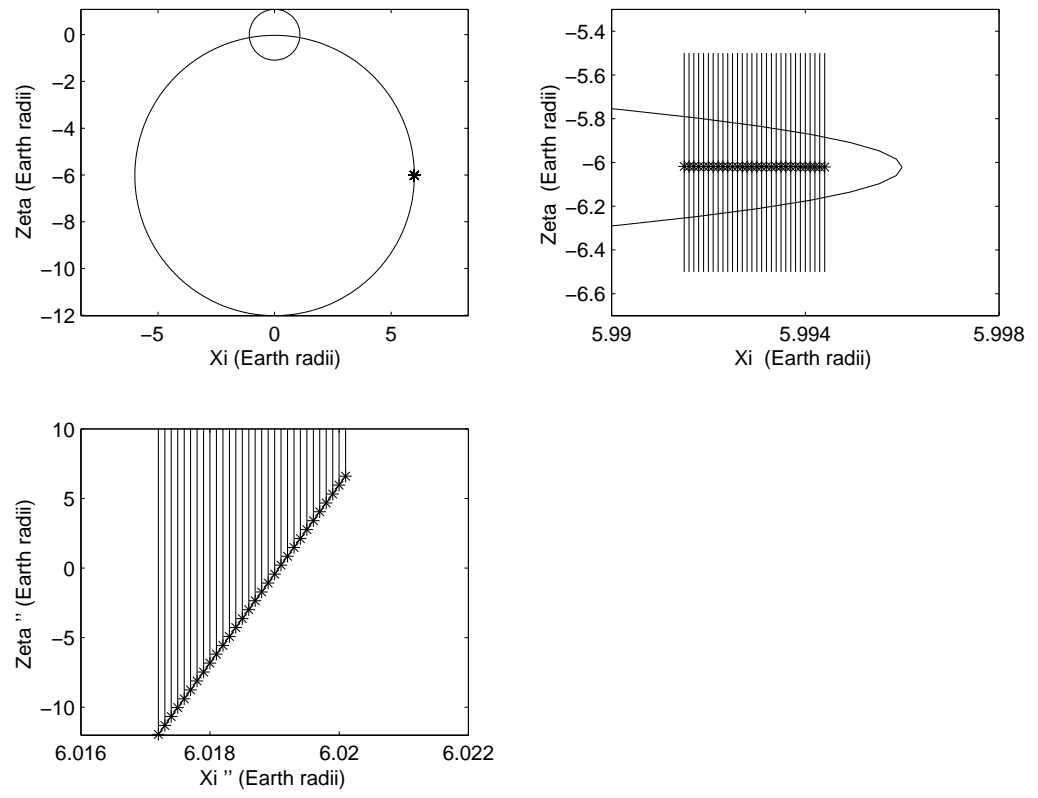

Fig. 3. Left: TP of the 2027 encounter of asteroid $1999 \mathrm{AN}_{10}$ with the Earth: the large circle corresponds to injection into the $3 / 5$ mean motion resonance, the small circle represents the Earth impact cross section. Right: an enlargement of the area inside the square on the left around the asterisk, showing VAs filling the region of the TP leading to an interrupted return in 2032. The fold points are marked with stars: as it is clear from the theory, the line of stars is inclined $-45^{\circ}$ with respect to the $\xi$ axis, but this is not visible because of the very unequal scale. Bottom: TP of the 2032 encounter for the VAs of the previous plot, showing the image of the fold (stars).

theory of returns and in the numerical experiments. In Figure 3 the image of a fold is obtained in an analytical way, by using the simple wire approximation on multiple parallel wires. The circle represents the exact $3 / 5$ resonance, corresponding to a return from the 2027 to the $2032 \mathrm{TP}$ (for the same node) of the asteroid $1999 \mathrm{AN}_{10}$.

Figures 4 and 5, on the contrary, were obtained by numerical integration. We have computed multiple solutions sampling the LOV. Then we have computed two "alternate nominal solutions" by moving along the direction of the eigenspace of the second largest eigenvalue of the covariance matrix, by an amount corresponding to $\sigma_{2}= \pm 2$, where $\sigma_{2}$ is the parameter along the "LOV2", analogous to the LOV but defined by the second eigenvalue. We have sampled with VAs the two "alternate LOVs" defined by using the alternate nominal solutions as initial conditions for the differential equation defining the ordinary LOV. We plot the corresponding TP points of all three LOVs.

In Figure 4 we see the image of a fold line, on the $2032 \mathrm{TP}$ of $2003 \mathrm{UM}_{3}$, with the folding towards the Earth, and indeed there is at least one VI, but it is hard to be confirmed with the algorithm discussed in Section 7 because 


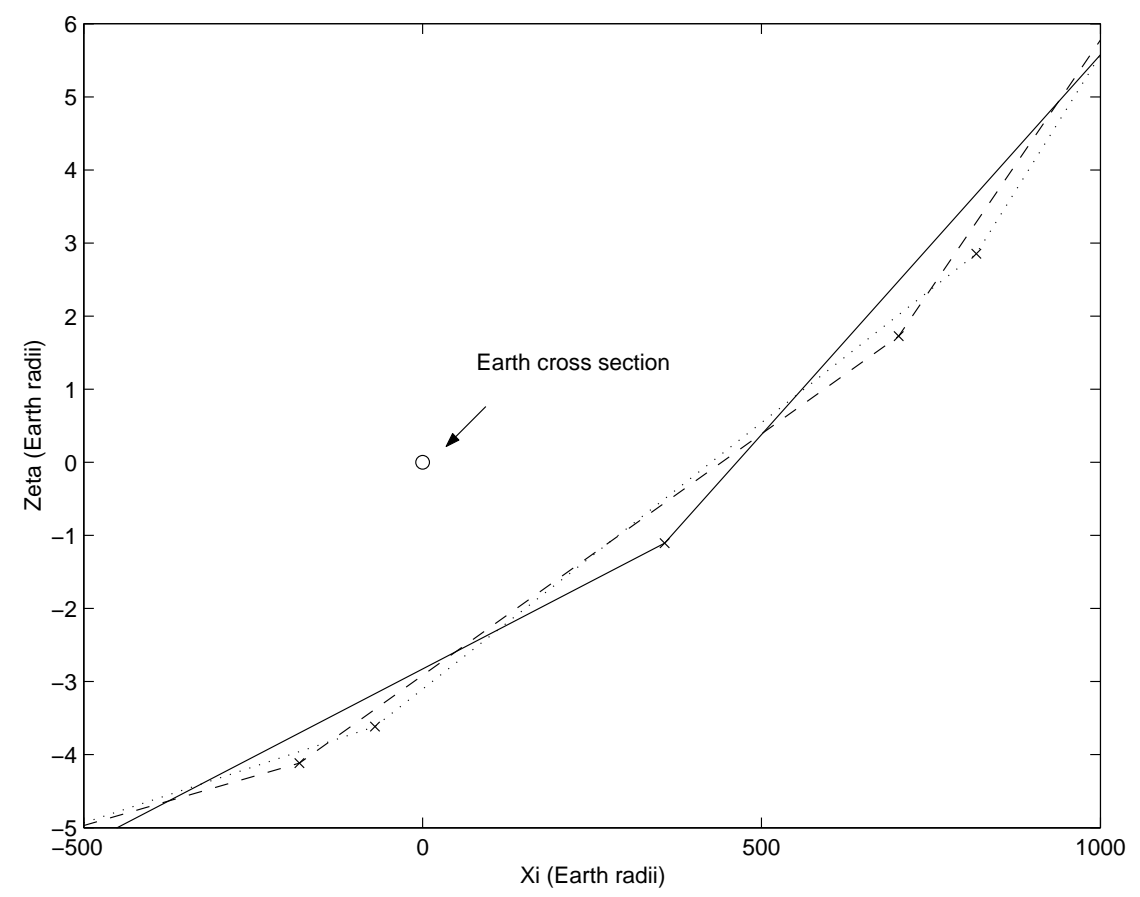

Fig. 4. For asteroid $2003 \mathrm{UM}_{3}$ a fold in the TP of the Earth in 2032. The full polygon represents a sampling of the nominal LOV, the dotted and the dashed ones are two "alternate LOV" on opposite sides of the nominal LOV, obtained with the procedure described in the text. In this case the fold is towards the Earth, thus the confidence region covers the Earth's impact cross section twice.

of the strong nonlinearity. Moreover, from the geometry we would expect to have two separate VIs, that is a disconnected set of initial conditions leading to an impact in 2032 , but our current algorithms cannot find both ${ }^{11}$.

Another fold is visible in Figure 5, on the $2032 \mathrm{TP}$ of the asteroid $1999 \mathrm{AN}_{10}$; this is the same case of the analytical computations shown in Figure 3. In this case the fold is away from the Earth, thus the point on the LOV of minimum distance is not close to an impacting initial condition, whatever the linearization may suggest. Of course the correspondence between the simplified picture given by the analytical theory and the numerical experiment is only qualitative: the real distribution of initial conditions inside the confidence region is much more complex than the wire approximation used for Figure 3.

At a cusp point, two folds come together and disappear; a normal form is

$$
\zeta^{\prime \prime}=\xi \zeta-\zeta^{3} \quad ; \quad \xi^{\prime \prime}=\xi
$$

This is also a generic behavior, but it occurs at isolated points on the first TP. However, it is not easy to find a clear example of cusp in the output of the

$\overline{{ }^{11} 2003} \mathrm{UM}_{3}$ has been observed only in two consecutive nights, and has been lost, being a very small object. 


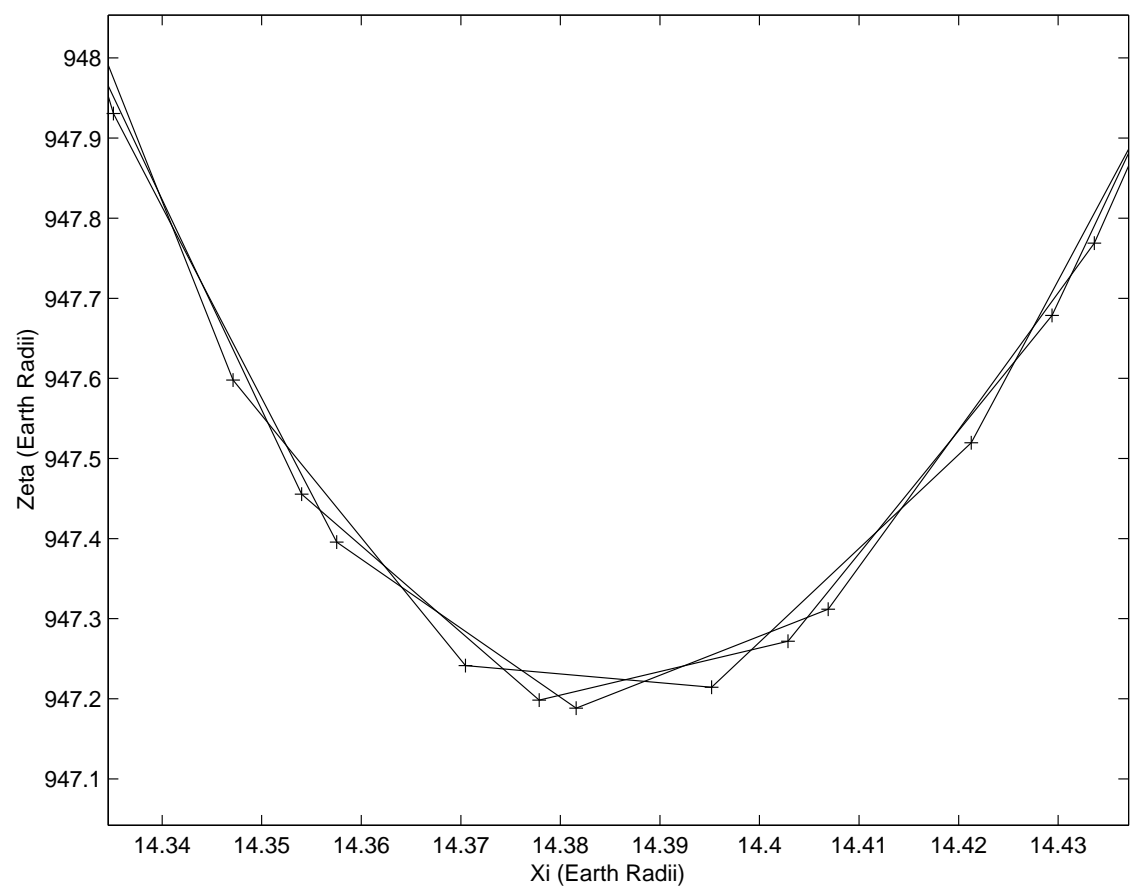

Fig. 5. For asteroid $1999 \mathrm{AN}_{10}$ a fold in the $\mathrm{TP}$ of the Earth in 2032. In this case the fold is in the direction opposite to the Earth, thus the confidence region does not map into the Earth's cross section. A linear TP analysis, given the semi-width of $\simeq 190$ Earth radii, would suggest the possibility of an approach closer than the ones found along the LOV, but in fact an approach at a distance lower than 940 radii is not compatible with the 101 observations used in this computation.

numerical integrations.

\section{GEOMETRY OF THE LOV TRACE ON THE TP}

\subsection{Decomposition into trails}

Operationally, the primary output of the propagation of the LOV is a collection of Earth encounters that have been detected for each VA during the time span of interest. This collection can be empty or it can be quite large; some objects have upwards of twenty encounters per VA. Initially, the encounter list is sorted in the way that it was obtained, that is, first by the VA index (i.e., by the LOV parameter $\sigma$ ) of the associated VA and then by time. But to conduct a coherent TP analysis, the encounters must be decomposed into dynamically related subsets that are clustered in both time and LOV $\sigma$.

The first step in the decomposition is to sort the encounters by date, followed by a splitting into showers that are isolated in time. A histogram of the en- 
counter dates typically reveals well-separated clusters situated near the times that the Earth passes the asteroid's nodes. Thus a shower represents all of the encounters occurring near a given node crossing in a given year. Next we need to subdivide the showers into contiguous LOV segments. To do this, the shower encounters are re-sorted according to their VA's LOV index, and then, advancing through the sorted list, we "cut" the shower wherever a gap in the LOV index is encountered. The trails thus obtained form sets of dynamically related encounters. Both CLOMON2 and Sentry use this algorithm to decompose the VA encounters into trails.

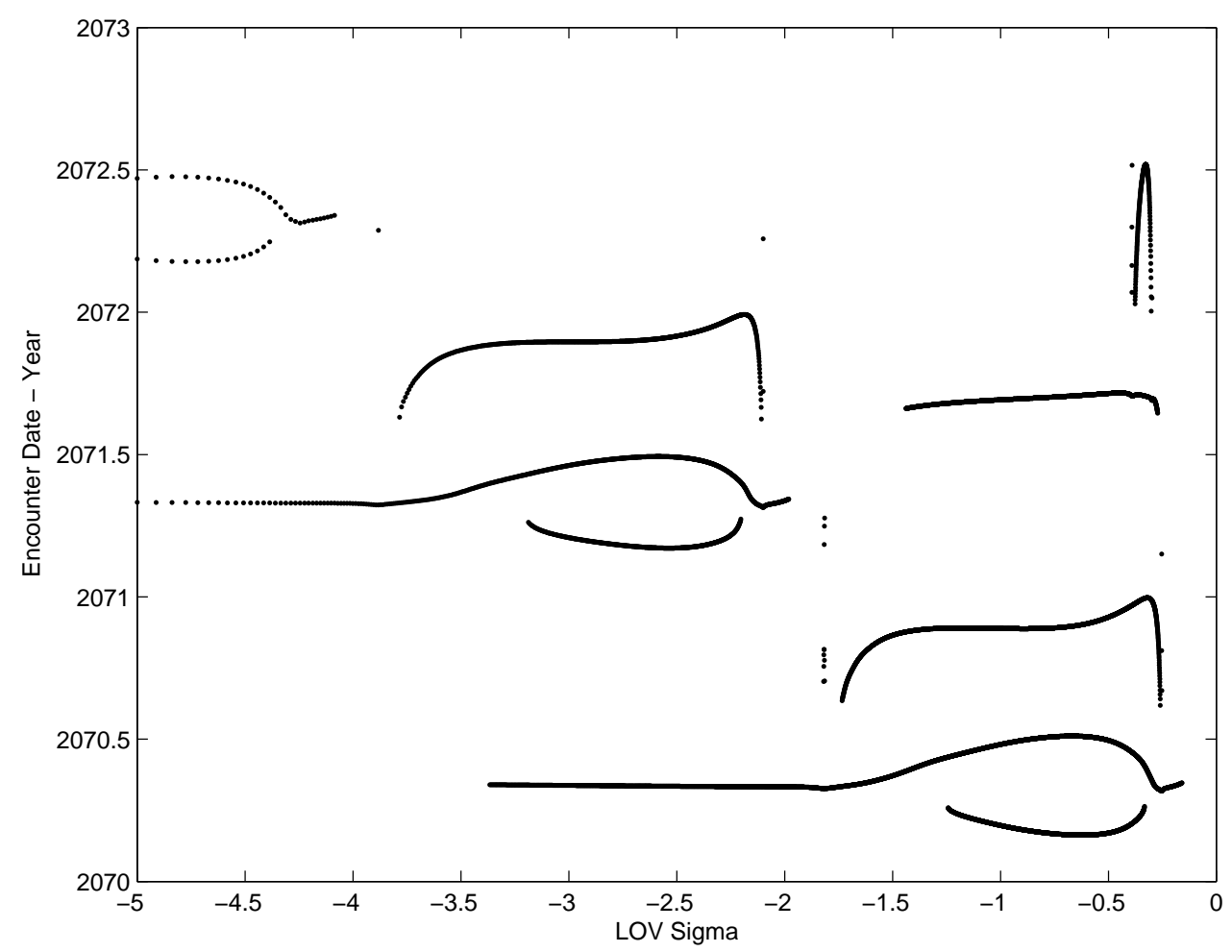

Fig. 6. Depiction of an extended shower for $2000 \mathrm{SG}_{344}$ occurring around 2070. The individual trails appear as separate loci of points in this depiction. Two VIs are present on a single trail in Sep. 2071 (2071.7) near LOV sigma -0.35.

The scheme described so far works well so long as the showers are well defined, but, in the case of Earth co-orbital asteroids, the orbit of the Earth and asteroid can be very close together for a large range of longitudes, even for all longitudes. In such cases, there may not be a clear clustering in time among the encounters. As an example, we consider $2000 \mathrm{SG}_{344}$, which has an Earthlike orbit with semimajor axis of $0.98 \mathrm{AU}$, eccentricity 0.07 and inclination $0.1^{\circ}$, leading to a relative velocity of only about $1.3 \mathrm{~km} / \mathrm{s}$. Figure 6 shows a single shower for $2000 \mathrm{SG}_{344}$ that continues without interruption for well over two years. The algorithm described so far treats this extended shower as a single trail despite the numerous and clearly distinct loci (trails) that are evident in the figure. 
Cases such as $2000 \mathrm{SG}_{344}$ need special treatment in order to isolate the individual trails. Sentry implements an additional splitting procedure whenever a trail is detected in which a single VA appears multiple times, as evidenced by duplicate LOV indices within the trail. Graphically, the procedure simply passes a vertical line across Fig. 6, all the while "filing" the encounters from distinct loci into separate trails. Computationally, the procedure steps through the shower according to increasing LOV index. At each LOV index, the encounters are assigned to the appropriate trail according to the encounter time. If the date of an encounter is inconsistent with one of the "open" trails then that encounter becomes the first in new trail. If an index advances without adding an encounter to a particular trail then that trail is "closed." This procedure helps to ensure that the individual trails comprise only closely related encounters, which is a key assumption in the TP analysis described below ${ }^{12}$.

\subsection{Geometry of the trails}

Our goal is to find VIs through a TP analysis; on this plane we know the trace $\vec{P}(\sigma)$ (corresponding to the value $\sigma$ of the LOV parameter) for a trail, that is a number of consecutive VAs used to sample the LOV. We will also use the corresponding derivative, the vector $\vec{S}(\sigma)=\partial \vec{P} / \partial \sigma(\sigma)$. When there are many points on the TP it is easy to understand the LOV behavior: in these cases the stretching is small and the linear theory is locally applicable. On the contrary, when there is a strong nonlinearity due to previous close approaches, the stretching is large and changes rapidly from point to point; in these situations the LOV behavior can be complex and a local analysis is necessary in the neighborhood of each VA. For this reason we have established the following geometrical classification of the possible LOV behaviors between two consecutive (in terms of multiple solutions index) VAs.

Let $\vec{P}_{1}$ and $\vec{P}_{2}$ be the geocentric position vectors on the TP of two consecutive VAs, corresponding to the values $\sigma_{1}, \sigma_{2}$ of the LOV parameter, respectively. Let $\vec{S}_{1}=\partial \vec{P}_{1} / \partial \sigma$ and $\vec{S}_{2}=\partial \vec{P}_{2} / \partial \sigma$ be the corresponding derivative vectors. Let $\alpha_{1}$ and $\alpha_{2}$ be the angles ang $\left(\overrightarrow{S_{1}}, \vec{\zeta}\right)$ and $\operatorname{ang}\left(\overrightarrow{S_{2}}, \vec{\zeta}\right)$ between the derivative vectors and the $\zeta$-axis (Fig. 7 ). We also set

$$
\begin{aligned}
\Delta \alpha & =\alpha_{1}-\alpha_{2} \\
\cos \beta_{1} & =\frac{\left(\vec{P}_{2}-\vec{P}_{1}\right) \cdot \vec{S}_{1}}{\left|\left(\vec{P}_{2}-\vec{P}_{1}\right)\right|\left|\vec{S}_{1}\right|}
\end{aligned}
$$

\footnotetext{
$\overline{{ }^{12} \text { Since }}$ complicated cases such as the one of Figure 6 are rare, they can be handled in CLOMON2 by manual intervention. Of course it would be desirable to implement an algorithm such as the one used by Sentry.
} 
$\cos \beta_{2}=\frac{\left(\vec{P}_{2}-\vec{P}_{1}\right) \cdot \vec{S}_{2}}{\left|\left(\vec{P}_{2}-\vec{P}_{1}\right)\right|\left|\vec{S}_{2}\right|}$

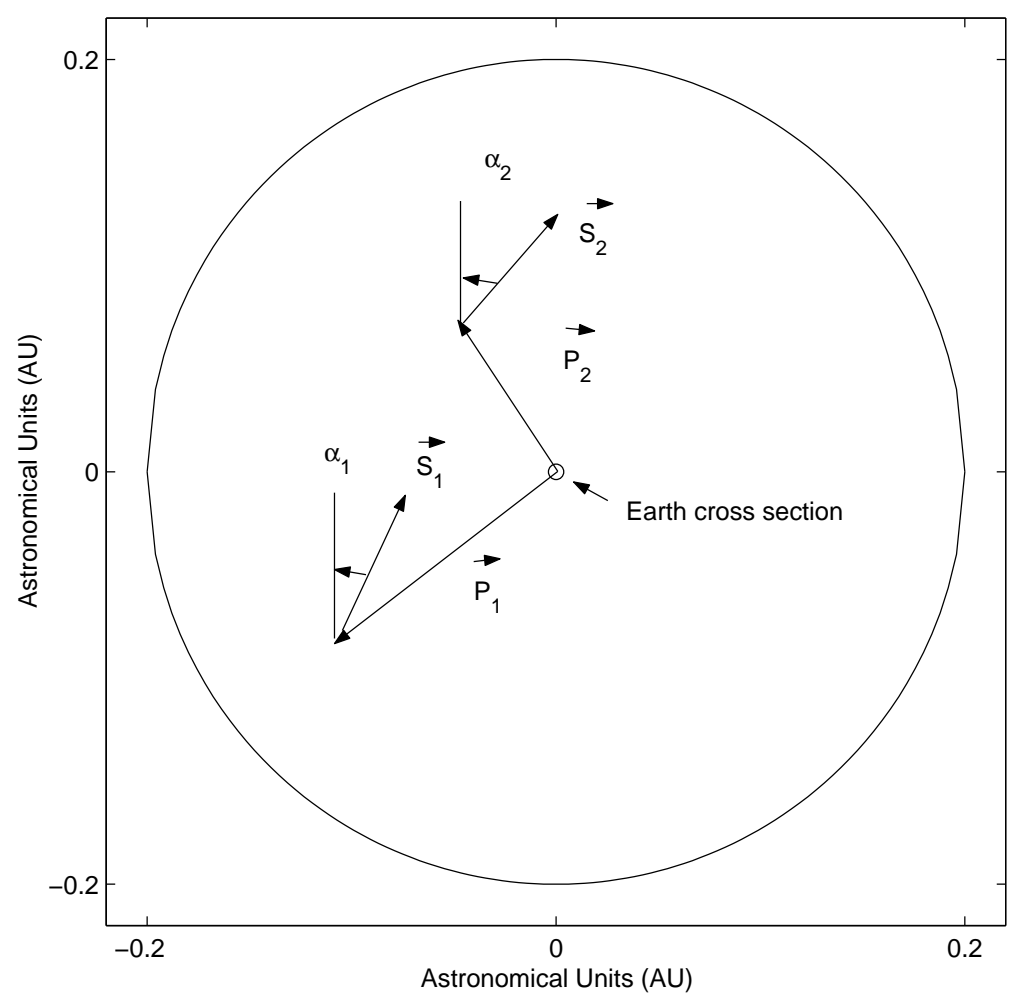

Fig. 7. The quantities used to classify the behavior of two consecutive TP points are obtained from the geocentric vectors $P_{i}$ and their derivatives (with respect to the LOV parameter) $S_{i}$.

A key function is the derivative of the square distance with respect to $\sigma$ :

$$
f(\sigma)=\frac{\partial r^{2}(\sigma)}{\partial \sigma}
$$

where $r^{2}(\sigma)=\xi^{2}(\sigma)+\zeta^{2}(\sigma)$ is the square distance of the VA from the center of the Earth (the origin of the TP reference system). The sign of $f(\sigma)$ provides critical information because it indicates the local increase and decrease of the distance.

For the classifications, we have taken into account:

(1) the angle $\Delta \alpha$ between the two derivative vectors;

(2) the sign of the function $f(\sigma)$ in $\vec{P}_{1}$ and $\vec{P}_{2}$.

(3) the alignment of the two derivative vectors with respect to the vector between the two TP points, through the angles $\beta_{1}$ and $\beta_{2}$;

We have identified six fundamental classifications of pairs of TP points based on criteria 1 and 2 above (Fig. 8), plus some special cases that use criterion 3 


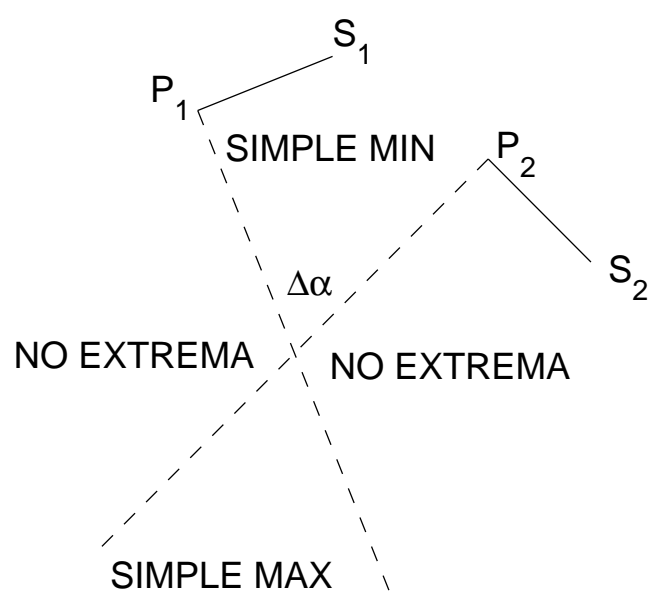

$\cos \Delta \alpha>0$

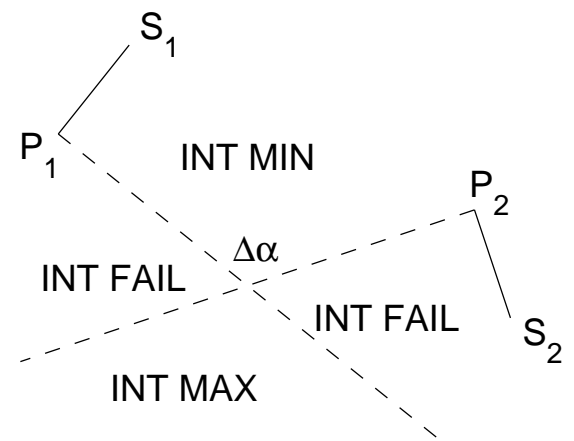

$\cos \Delta \alpha<0$

Fig. 8. Schematic depiction of the six fundamental classifications of pairs of points on the LOV, for simple $(\cos \Delta \alpha>0$, on the left) and reversing $(\cos \Delta \alpha<0$, on the right) returns. The classification is obtained according to the location of the Earth with respect to the locations and orientations of $\vec{S}_{1}$ and $\vec{S}_{2}$, according to the regions marked on the figure.

(Tommei 2002). All are listed in Table 1 and described below.

\begin{tabular}{lccc} 
& $\cos \Delta \alpha$ & $f\left(\sigma_{1}\right) \cdot f\left(\sigma_{2}\right)$ & $f\left(\sigma_{2}\right)$ \\
\hline SIMPLE MIN & $>0$ & $<0$ & $>0$ \\
SIMPLE MAX & $>0$ & $<0$ & $<0$ \\
NO EXTREMA & $>0$ & $>0$ & - \\
INT MIN & $<0$ & $<0$ & $>0$ \\
INT MAX & $<0$ & $<0$ & $<0$ \\
INT FAIL & $<0$ & $>0$ & - \\
ENTANGLED & $>0$ & - & - \\
UNRELATED & $>0$ & $<0$ & - \\
\hline$\beta_{1} \mid \geq \sin \beta_{\text {lim }}$ and & \multicolumn{2}{c}{$\sin \beta_{2} \mid \geq \sin \beta_{\text {lim }}}$. \\
. CLOMON2 only.)
\end{tabular}

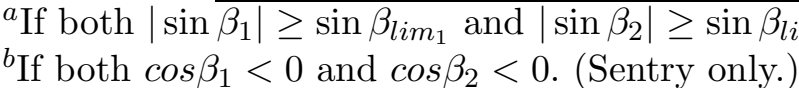

Table 1

Classification of LOV segments on the TP between two consecutive VAs. The six fundamental types (Fig. 7) are listed first, followed by special cases that are unique to either CLOMON2 or Sentry.

The first three classifications have the property that the curvature of the LOV trace on the TP between the two points is moderate, at most $90^{\circ}$. 
- SIMPLE MINimum ${ }^{13} f\left(\sigma_{1}\right)<0$ and $f\left(\sigma_{2}\right)>0$; thus in $\left[\sigma_{1}, \sigma_{2}\right]$ there is at least one point $\left(\sigma=\sigma_{\text {min }}\right)$ with $f\left(\sigma_{\text {min }}\right)=0$ and which is a minimum for the distance from the Earth.

- SIMPLE MAXimum $f\left(\sigma_{1}\right)>0$ and $f\left(\sigma_{2}\right)<0$; thus in $\left[\sigma_{1}, \sigma_{2}\right]$ there is at least one maximum in the distance from Earth. This case is ignored under the hypothesis that in the interval is present only one maximum (see the comment at the end of section).

- NO EXTREMA Both points are going away or both are approaching to the Earth, but the positions of $\vec{S}_{1}$ and $\vec{S}_{2}$ do not indicate the presence of extrema of the function $r^{2}(\sigma)$ in $\left[\sigma_{1}, \sigma_{2}\right]$. Also this case is ignored.

The next three classifications indicate a substantial curvature, generally a reversal, of the LOV between the two points $\vec{P}_{1}$ and $\vec{P}_{2}$.

- INTerrupted MINimum It is an interrupted return. The LOV seems to behave as in a SIMPLE MIN, but, for some value $\sigma$, it turns back and it goes away in the opposite direction. The tip of this interrupted can be before the Earth (the function $r^{2}(\sigma)$ has only one minimum), beyond the Earth (the function $r^{2}(\sigma)$ has two points of minimum and one point of maximum) or even inside the Earth.

- INTerrupted MAXimum Opposite to INT MIN, in $\left[\sigma_{1}, \sigma_{2}\right]$ there is at least one maximum. Also this case is ignored.

- INTerrupted FAILed It is a part of interrupted with maximum: in $\left[\sigma_{1}, \sigma_{2}\right]$ there at least one maximum and one minimum.

There are a few special cases that are only considered by one of the two monitoring systems; they are detected according to the values of the angles $\beta_{1,2}$ as described in Table 1.

- ENTANGLED (If $\cos \Delta \alpha>0$ and both $\left|\sin \beta_{1}\right| \geq \sin \beta_{l i m_{1}}$ and $\left|\sin \beta_{2}\right| \geq$ $\sin \beta_{\text {lim }_{2}}$. CLOMON2 only.) This is the most complicated case to be handled. The segment of the LOV between $P_{1}$ and $P_{2}$ passes either two or three times near the Earth, and so in $\left[\sigma_{1}, \sigma_{2}\right]$ the function $f$ has at least four zeroes corresponding to two maxima and two minima in the distance from the Earth (three minima can also occur).

A tricky issue for the ENTANGLED classification is the choice of limit values for $\sin \beta_{i}(i=1,2)$. A constant value would not be satisfactory, because it would not take into consideration the changes in the orientation of the TP resulting from the motion of the Earth on its own orbit; in fact the TP is not the same for all VAs, but it undergoes small changes from one to another. The limit value for $\sin \beta_{i}$ must depend upon the distance and

\footnotetext{
$\overline{13}$ This notation puts in evidence the shortened name used in the table.
} 


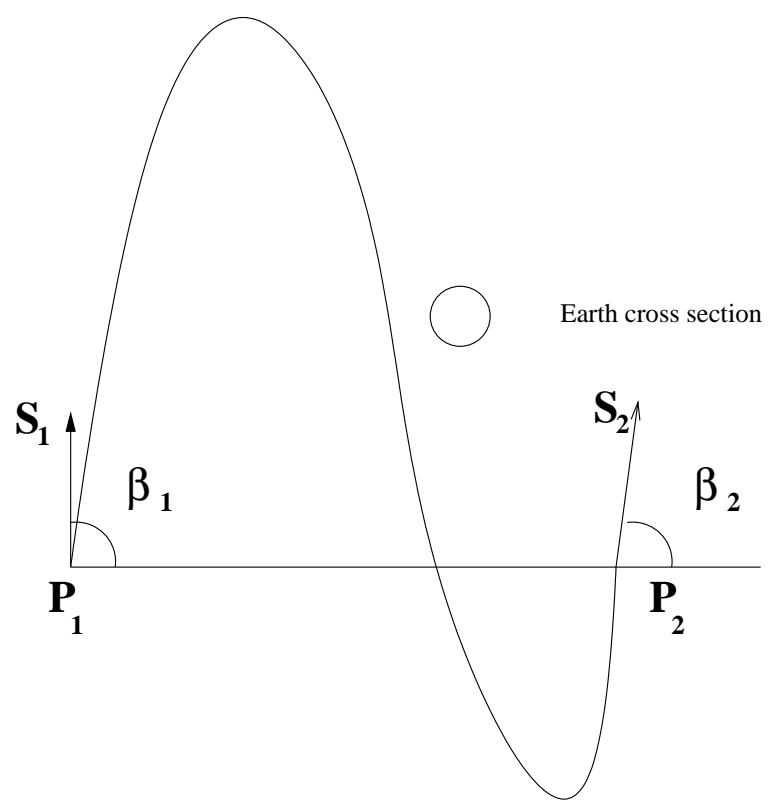

Fig. 9. The angles $\beta_{1}$ and $\beta_{2}$ describe the direction of the LOV tangent with respect to the line joining two consecutive TP points. They are used in the definition of the ENTANGLED and UNRELATED types. The Figure depicts schematically one of the possible ENTANGLED geometries, the one with two minima and two maxima between $P_{1}$ and $P_{2}$, the first stationary point after $P_{1}$ being a minimum.

the velocity of the VA:

$$
\sin \beta_{\lim _{i}}=\max \left(k_{s} k \frac{r_{i}}{U_{i}}, \sin 20^{\circ}\right)
$$

where $k=\frac{2 \pi}{365}$ is the Gauss constant, that is the angular velocity of the Earth expressed in radians per day, while $k_{s}$ is a security factor (e.g., we use $\left.k_{s}=4\right)$.

- UNRELATED (If both $\cos \beta_{1}<0$ and $\cos \beta_{2}<0$. Sentry only.) The LOV direction, as given by the two consecutive TP points is more than $90^{\circ}$ different from the LOV direction indicated by both derivative vector. Experiments have shown that such TP points are often unrelated, that is they have had a different sequence of previous encounters, and even if they are related, LOV nonlinearities effectively limit the IP of associated VIs. Thus the pair can be safely ignored; this is justified for Sentry due to the increased sampling density along the LOV.

Finally, there are three additional cases in which individual VAs, rather than pairs, are considered:

- HEAD It is the first VA of a trail with $f(\sigma)>0$

- TAIL It is the last VA of a trail with $f(\sigma)<0$

- SINGLETON There is only one VA on the TP (in the trail). Note that a SINGLETON is also either a HEAD or a TAIL. 


\subsection{The principle of the simplest geometry}

The classification we have presented uses the hypothesis that the LOV follows, among paths which satisfy certain geometrical conditions, the most simple. Every close approach introduces nonlinearity, that, on the TP of the subsequent encounter, is transformed into a geometrically complex LOV curve (see Figure 1). Thus it is possible that, in the short segment of the LOV parameterized by $\left[\sigma_{1}, \sigma_{2}\right]$, there are many stationary points of the function $r^{2}(\sigma)$. To obtain a finite procedure we need to introduce a limitation to the geometrical complexities that we explore. We need to stress once again that the purpose of our monitoring system is to find some of the VIs, not necessarily all of them. Moreover, it could be argued that the additional VIs which could be found in TP geometries of the LOV more complex than the minimum would correspond to higher values of the stretching, thus should have substantially lower impact probabilities than the generic completion level discussed earlier. However, it has to be acknowledged that this is only a qualitative argument for which we are not able to provide a quantitative estimate.

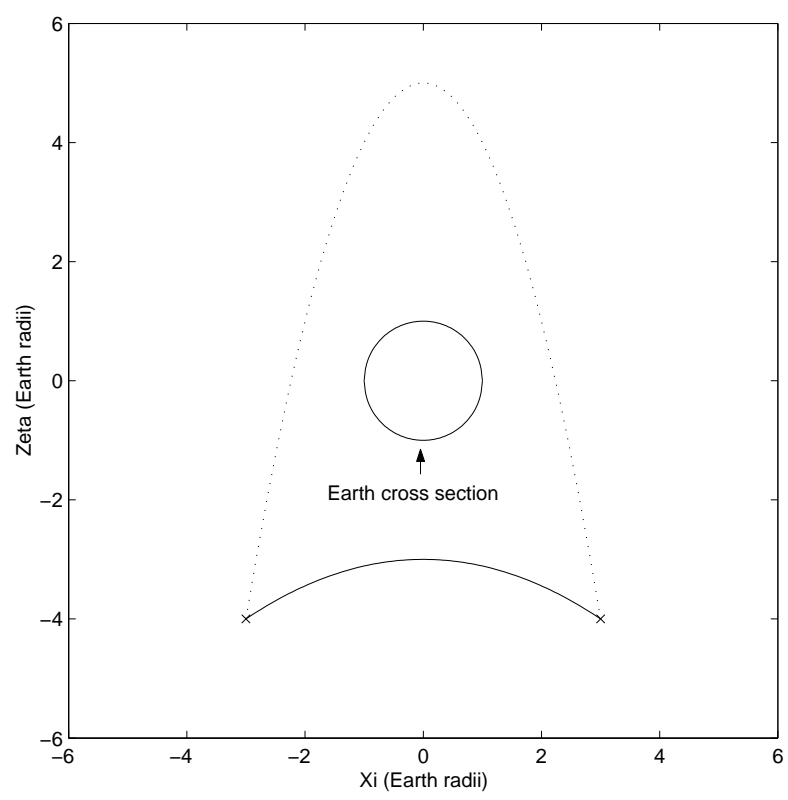

Fig. 10. In the interrupted minimum case, there are two possible interpretations of the geometry of the LOV segment between two consecutive points, both satisfying the principle of the simplest geometry. There could be either an interrupted minimum as the only stationary point (continuous line) or an interrupted maximum and two simple minima (dotted line).

There is one case of complexity in the distribution of minima of $r^{2}(\sigma)$ which does occur comparatively frequently, does not introduce additional complexity in the geometry and can result in additional VIs which are not necessarily less likely than the others. As discussed above in the definition of the INT MIN case (see Figure 10) the tip of the interrupted return can be either "before" 
or "after" the Earth. CLOMON2 and Sentry solve this problem in different ways, as we shall see in the following section.

\section{ONE DIMENSIONAL DISTANCE MINIMIZATION}

The LOV approach is very favorable for local analysis because the VAs belong to a continuous one-dimensional set and it is possible to interpolate between them. When we examine a trail we check if the minimum distance to the Earth across the LOV could be small, by computing the quantity $d_{\min }=$ MOID $-\kappa w$, where $w$ is the semi-width of the 2-dimensional confidence region, as computed in the linear TP analysis (Milani et al. 2002). (Sentry uses $\kappa=5$ and CLOMON2 uses $\kappa=3$.) If $d_{\text {min }}$ is small enough to allow an impact then we continue our analysis with iterative schemes to determine the minimum distance.

The basic algorithm used by both Sentry and CLOMON2 is the modified regula falsi. In some cases, CLOMON2 also uses an alternative approach, which we call Newton's method with bounded step. Whichever method is used, the objective is to find a zero of the function

$$
f(\sigma)=\frac{\partial r^{2}(\sigma)}{\partial \sigma}
$$

in an interval $\left[\sigma_{1}, \sigma_{2}\right]$.

\subsection{MODIFIED REGULA FALSI}

Let $f(x) \in C^{0}\left[a_{0}, b_{0}\right]$ be a continuous function with $f\left(a_{0}\right) f\left(b_{0}\right)<0$. Let $F=$ $f\left(a_{0}\right), G=f\left(b_{0}\right)$ and $w_{0}=a_{0}$. We use a modification of the classical regula falsi method (Conte and de Boor, 1972), which proceeds as follows. For $n=0,1,2, .$. until convergence:

- compute $w_{n+1}=\left(G a_{n}-F b_{n}\right) /(G-F)$

- if $f\left(a_{n}\right) f\left(w_{n+1}\right) \leq 0$, we put $a_{n+1}=a_{n}, b_{n+1}=w_{n+1}, G=f\left(w_{n+1}\right)$

- if moreover $f\left(w_{n}\right) f\left(w_{n+1}\right)>0$, we put $F=F / 2$

- otherwise, we put $a_{n+1}=w_{n+1}, b_{n+1}=b_{n}, F=f\left(w_{n+1}\right)$

- if moreover $f\left(w_{n}\right) f\left(w_{n+1}\right)>0$, we put $G=G / 2$

If convergent, the algorithm finds a zero of $f(x)$ in $\left[a_{n+1}, b_{n+1}\right]$. Of course, the algorithm is surely convergent if the function $f$ is defined and continuous in the interval $\left[\sigma_{1}, \sigma_{2}\right]$, but speed of convergence can be a problem. Failure can occur if for some value of $\sigma$ in the interval the function is undefined, that is, 
the TP of the encounter around that date is missed, which generally indicates that the two TP points under consideration are not part of the same trail. Of course we also need to fix a maximum number of iterations of the regula falsi, thus there can be another case of failure: a lack of convergence to the required accuracy within the maximum number of iterations.

To reduce the number of cases of slow convergence, CLOMON2 has implemented a further accelerated version of the modified regula falsi described above. Specifically, the algorithm is the same, but the value of the function is divided by 4 when $f\left(w_{n}\right) f\left(w_{n+1}\right)>0$. This variant is used when the convergence is slow, that is when the value of the function remains of the same sign for three or more iterations.

The Sentry convergence criteria is that the TP separation of the two regula falsi endpoints must be less than a tenth of the Earth's radius. A lack of convergence is extremely unusual for Sentry, presumably due to the use of much smaller LOV steps. Those cases that do fail to converge have usually been associated with unrelated pairs of TP points, violating one of the fundamental hypotheses of regula falsi.

\subsection{NEWTON METHOD WITH BOUNDED STEP}

When it is necessary or desirable to minimize the distance by starting with only a single TP point, we apply a variant of Newton's method of root-solving. (The following method is used by CLOMON2 only, as Sentry ignores Singleton trails.)

To begin, let $\vec{P}$ be the coordinate vector of the point on the LOV, in the reference system of the TP:

$$
\vec{P}=(\xi, \zeta)
$$

Let $r$ be the distance between the asteroid and the center of the Earth on the TP and let $\alpha$ be the angle between the derivative vector $\vec{S}$ and the $\zeta$ axis. Since $r^{2}=\vec{P} \cdot \vec{P}$ and $\vec{S}=\Lambda(-\sin \alpha, \cos \alpha)$, with $\Lambda=|\vec{S}|$ the stretching.

The function under analysis to find zeroes is

$$
f=\frac{\partial r^{2}}{\partial \sigma}=2 \vec{P} \cdot \frac{\partial \vec{P}}{\partial \sigma}=2 \vec{P} \cdot \vec{S}=2 \Lambda(-\xi \sin \alpha+\zeta \cos \alpha)
$$


The pseudo-Newton method iteration is then

$$
\sigma_{i+1}=\sigma_{i}-\frac{f}{\tilde{g}}
$$

where $\tilde{g}=2 \Lambda^{2}$ is an approximation of the second derivative $g$ of $r^{2}(\sigma)$

$$
g=\frac{\partial f}{\partial \sigma}=\frac{\partial^{2} r^{2}}{\partial \sigma^{2}}=2\left(\vec{S} \cdot \vec{S}+Y \cdot \frac{\partial \vec{S}}{\partial \sigma}\right)
$$

$\tilde{g}$ is obtained by neglecting $\frac{\partial \vec{S}}{\partial \sigma}$, that is by considering that the trace of the LOV on the TP is just a line segment parameterized by $\sigma$ (in particular, the stretching $\Lambda$ is considered constant).

However, unlike the classical pseudo-Newton method, the step, at each iteration, is compared with an upper bound, $\left(\sigma_{l i m}\right)$, and we control the step size of the successive iterations in such a way that the sequence $\sigma_{i}$ is within the interval $\left[\sigma_{0}-\sigma_{\text {lim }}, \sigma_{0}+\sigma_{\text {lim }}\right]$. After the $n$-th iteration we use a correction such that its absolute value is not larger than $\sigma_{l i m} / 2^{n}$. Remembering the celebrated paradox of Zeno (Achilles and the turtle), the total accumulated change in $\sigma$ cannot exceed $\sigma_{\text {lim }}$. Thus this method cannot diverge, but can fail to converge in only two ways: either by finding a value of $\sigma$ for which the TP is missed, or by performing more than a preset maximum number of iterations without achieving convergence with the required accuracy.

\subsection{METHODS APPROPRIATE TO EACH GEOMETRY}

For each of the geometries described in Section 4 we need to select, among the minimization algorithms of this Section, the most appropriate one. It is clear that regula falsi is specific for the SIMPLE MIN case, while the bounded Newton method is the only option for the SINGLETON case. However, we need to select a method for all cases! Table 2 describes the action taken by each system for the various geometric classifications. "Skip" means that by the principle of simplest geometry we need not to assume the presence of a LOV minimum. "Ignore" indicates that Sentry ignores these cases under the assumption that the impact probability of any detected VI will be around or below its threshold of interest.

Both Sentry and CLOMON2 apply some method to find the minimum distance along the LOV when the geometric configuration belongs to the cases indicated in the Table and at the same time the linearized theory indicates that a very close approach is possible. 


\begin{tabular}{lcc} 
& CLOMON2 & Sentry \\
\hline SIMPLE MIN & Regula Falsi & Regula Falsi \\
SIMPLE MAX & Skip & Skip \\
NO EXTREMA & Skip & Skip \\
INT MIN & Regula Falsi + Newton & LOV Densification \\
INT MAX & Skip & Skip \\
INT FAIL & Newton & LOV Densification \\
ENTANGLED & Newton & - \\
UNRELATED & - & Skip \\
SINGLETON & Newton & Ignore \\
HEAD & Newton & Ignore \\
TAIL & Newton & Ignore \\
\hline
\end{tabular}

Table 2

The action taken by each monitoring system for the various TP classifications.

As is clear from Table 2, Sentry makes use of its denser LOV sampling by completely ignoring the SINGLETON, HEAD/TAIL and UNRELATED cases which cannot lead to impact probabilities greater than the generic completion level. On the other hand, the INT MIN and INT FAIL cases must be considered. Sentry approaches these situations with $L O V$ densification, i.e., sampling the LOV between $\sigma_{1}$ and $\sigma_{2}$ with much smaller steps. (At present Sentry subdivides the interval into 20 subintervals, and so the integral probability between oversampled points is $5 \times 10^{-6}$.) Next a kind of recursion is applied, whereby the consecutive pairs of TP points obtained by LOV densification are classified according to Table 1. Only SIMPLE MIN cases are analyzed further after LOV densification; other classifications are ignored.

For CLOMON2, the INT MIN case is especially challenging because we cannot know, from the information provided by two TP points only, whether it is a true interrupted return or a failed interrupted return, with two minima and one maximum (see Figure 10). Thus, after finding one minimum with regula falsi, it is necessary to start from the extremum which could be closer to the other minimum with a Newton's method. This is determined from whether the local minimum found has an LOV tangent aligned with either the point $P_{1}$ or with the point $P_{2}$. A new search procedure for an additional minimum can then be started from the other one of these two points, the one less aligned. Of course if the return is really interrupted, that is there is a single minimum, the additional search will end up by finding the same minimum again, and this duplicate has to be removed from the output. 
The use of Newton's method enables an additional technique that improves the reliability of the iterative process. When, in the sequence of iterations, two consecutive TP points are found with values of the function $f$ having opposite sign, it is possible to identify either a SIMPLE MIN or a INT MIN case, and to switch to the regula falsi applied to the smaller segment.

The ENTANGLED cases do not allow for a single minimum in the interval, even assuming the simplest geometry. The current procedure finds only one of the minima. If the goal was to find all the minima, an alternate procedure, such as the LOV densification used by Sentry, would be needed.

\section{ESTIMATION OF IMPACT PROBABILITY}

If a VI has been found along the axis of the TP confidence region, and the semi-width of this region is much smaller than the diameter of the Earth, it is possible to estimate the Impact Probability (IP) with a very simple algorithm (see Milani et al. 2002 for more details).

When the width of the confidence region is not negligible we have to compute a 2-dimensional probability integral on the TP. We use a Gaussian probability distribution; in particular, given a trace on the $\mathrm{TP} \vec{P}(\sigma)=(\xi, \zeta)$ with stretching $\Lambda$ and semi-width $w$, the probability density function is more easily expressed by performing first a rotation of the coordinates by the angle $-\alpha_{\Lambda}$ between the major axis of the confidence ellipse on the TP and the $\zeta$ axis. If $\left(\xi^{\prime}, \zeta^{\prime}\right)$ are the rotated coordinates, the probability density is the product of the two densities

- $\rho_{1}(u)=\frac{1}{\sqrt{2 \pi} w} \exp \left[-\frac{1}{2}\left(\frac{u-\xi^{\prime}}{w}\right)^{2}\right]$, along the width direction,

- $\rho_{2}(v)=\frac{1}{\sqrt{2 \pi} \Lambda} \exp \left[-\frac{1}{2}\left(\frac{v-\zeta^{\prime}}{\Lambda}+\sigma\right)^{2}\right]$, along the stretching direction.

Then the complete probability density is

$$
\rho(u, v)=\frac{1}{2 \pi w \Lambda} \exp \left\{-\frac{1}{2}\left[\left(\frac{u-\xi^{\prime}}{w}\right)^{2}+\left(\frac{v-\zeta^{\prime}}{\Lambda}+\sigma\right)^{2}\right]\right\},
$$

and the probability of an Earth impact is

$$
I P=\iint_{D_{\oplus}} \rho(u, v) d v d u
$$

where $D_{\oplus}$ is the impact cross section on the TP (note that the disk has a radius $b_{\oplus}$, larger than the physical radius of the Earth $R_{\oplus}$, because of gravitational 
focusing). In practice it is not necessary to compute this integral where the probability density function is negligible, e.g., farther from the LOV trace on the TP by more than $8 w$. Thus the integral can be computed as an integral over a finite TP domain:

$$
I P=\int_{r_{\min }}^{r_{\max }} d u \int_{-\sqrt{b_{\oplus}^{2}-u^{2}}}^{\sqrt{b_{\oplus}^{2}-u^{2}}} \rho(u, v) d v
$$

where $r_{\max }=\min \left(b_{\oplus}, \xi+8 w\right), r_{\min }=\max \left(-b_{\oplus}, \xi-8 w\right)$

\section{APPLICABILITY OF LINEARIZATION}

It is not always possible to fit all cases into the scheme seen in the previous section; nonlinearity plays an important role in close approaches, so phenomena such as interrupted returns or the " $Z$ " shapes of Figure 2 appear. Our method, which consists in a linearization in a neighborhood of a VA on TP, works quite well also in situations with high stretching, excluding the case in which the impact takes place near a fold point (the tip of an interrupted). In these situations the stretching is very low, while the semi-width of the linearized confidence region around the point is quite large.

When the impact probability is computed, the probability density on the TP decreases exponentially in the direction orthogonal to the LOV; the exponent is inversely proportional to the semi-width $w$. Thus for large $w$ it is possible to have significant probability values even when the minimum distance along the LOV is large. Near the tip of an interrupted return the large value of $w$ indicates the nonlinearity of the situation rather than a large confidence strip on the TP, thus we can expect that a fully nonlinear probability computation would give a very different result. This could result in a spurious VI, by definition a connected region of the initial conditions space which appears to lead to a collision only because of some linearization, but there is no nearby collision orbit.

CLOMON2 and Sentry have taken very different approaches in dealing with this problem, as we describe in the following sections. This is currently the most important difference between the two systems, and this lack of convergence reflects the fact that neither of the two has found a procedure working in all cases. 


\subsection{CLOMON2 Spurious VI Filtering}

To eliminate VIs, CLOMON2 applies a "VI verification algorithm" based on the principle that a potential VI is real if the actual impacting initial conditions can be found. Such initial conditions are called a representative of the VI. Thus, starting from the orbit corresponding to the minimum distance along the LOV, as found with the algorithms of Section 5, we try to find a small change in the initial conditions leading to an actual impact. This is obtained with a modification of the algorithm introduced in Milani et al. (2000a).

Let $X_{j}$ be the initial condition corresponding to the minimum approach distance along the LOV, and $F$ the map from the initial conditions space to the $\mathrm{TP}$ of the encounter. The correction sought on the TP is

$$
\Delta T_{\text {target }}=T_{\text {min }}-F\left(X_{j}\right), \quad F: X \longmapsto T
$$

where $T_{\min }$ is the point, on the disk of the Earth with radius $b_{\oplus}$, of minimum distance from $F\left(X_{j}\right)$. In the linear approximation the orbital elements variation $\Delta X=X-X_{j}$ such that

$$
D F \Delta X=\Delta T_{\text {target }}
$$

would satisfy the target requirement. To compute $\Delta X$ we consider $D F$ as the composition of a projection $\Pi_{E}$ on a two-dimensional subspace $E$ with a bijective map $A: E \rightarrow T$ :

$$
D F=A \circ \Pi_{E} .
$$

where $E$ is a 2-dimension subspace of $X$ spanned by rows of the matrix $D F$. Then we define $L$ as the 4-dimension subspace orthogonal $E$, in such a way that the couple $(E, L)$ parameterizes $X$. Then it is possible to change coordinates for $X$ by means of an orthogonal matrix $V(6 \times 6)$ such that:

$$
\Delta X=V\left(\begin{array}{c}
\Delta E \\
\Delta L
\end{array}\right)
$$

with $\Delta E$ in the $E$ subspace and $\Delta L$ in the $L$ subspace. In the new reference system the normal matrix $C$ is

$$
V^{T} C V=\left(\begin{array}{cc}
C_{E} & C_{E L} \\
C_{L E} & C_{L}
\end{array}\right) .
$$

In the linear approximation, an orbital elements variation along $L$ does not change the position on the TP: the projection $\Pi_{E}$ is not invertible because 
the pre-image of each point is a 4-dimensional space. On the contrary, all the variations along $E$ are mapped on the TP in a bijective way, thus there is an inverse map $B=A^{-1}$.

However it is possible to select in an optimal way a $\Delta X$ with a fixed image $\Delta T$ on TP. The part of pre-image contained in the confidence ellipsoid can be described by the inequality

$$
\begin{gathered}
\sigma^{2} \geq \Delta X^{T} C \Delta X= \\
\Delta L^{T} C_{L} \Delta L+2 \Delta L^{T} C_{L E} \Delta E+\Delta E^{T} C_{E} \Delta E= \\
\left(\Delta L-L_{0}\right)^{T} C_{L}\left(\Delta L-L_{0}\right)+\text { const }
\end{gathered}
$$

where $\Delta E=B \Delta T$ is fixed, unequivocally determined by the displacement $\Delta T$. The inequality, with only $\Delta L$ as variable, describes a 4 -dimensional ellipsoid (with its interior part) $Z(\Delta T)$; therefore it is possible to select the point $L_{0}$, symmetry center of the quadric, as representative of $Z(\Delta T)$. Note that, in the linear approximation, $L_{0}$ corresponds to the minimum of the target function among the points mapping onto $\Delta T$. $L_{0}$ can be computed as function of $\Delta E$ :

$$
L_{0}=-C_{L}^{-1} C_{L E} \Delta E .
$$

In this way we are able to choose

$$
\Delta X=V\left(\begin{array}{c}
\Delta E \\
-C_{L}^{-1} C_{L E} \Delta E
\end{array}\right)=V\left(\begin{array}{c}
I_{2} \\
-C_{L}^{-1} C_{L E}
\end{array}\right) B \Delta T=H(\Delta T)
$$

in such way that (1) is fulfilled.

The next step is to propagate the orbit with initial conditions $X_{j}+\Delta X$ until the time of close approach; because of the nonlinear effects,

$$
F\left(X_{j}+\Delta X\right) \neq F\left(X_{j}\right)+\Delta T
$$

but the distance between the new point on the TP and $T_{\min }$ could be smaller, if the nonlinearity is mild. This correction can be iterated, like in the usual Newton's method: we put the nominal orbit at $X_{j}+\Delta X$, we calculate its TP trace and its partial derivatives matrix $D F$, then we choose a new $\Delta X$ fulfilling the new version of the equation (1), and so on, until convergence. If the close approach distance, calculated at convergence, is less than the Earth impact parameter, we have found a representative of the Virtual Impactor.

As is always the case with Newton's method, convergence is not guaranteed. We are using a number of criteria to terminate the iterations of the correction 
step, and in each of them we claim that the VI found by linearization from a LOV point is spurious. In fact, the rigorous statement should be that we have not found a confirmed VI, since we have not been able to explicitly compute a representative.

The criteria for terminating the Newton iterations are the following:

(1) The scalar product between $\Delta T_{\text {target }}$ and $\Delta T_{\text {found }}$, that is between the displacement we wanted and the actual displacement found on the TP, is negative. The point we have found has moved in the opposite direction on the TP, and this can indicate that a fold is present (see Figure 11).

(2) The RMS value of the residuals, computed at the collision solution, is too large.

(3) One iteration provides initial conditions such that the orbit does not cross the TP (within a radius of $0.2 \mathrm{AU}$ ) at times close to the one of the other close approaches in the same shower (see Figure 11).

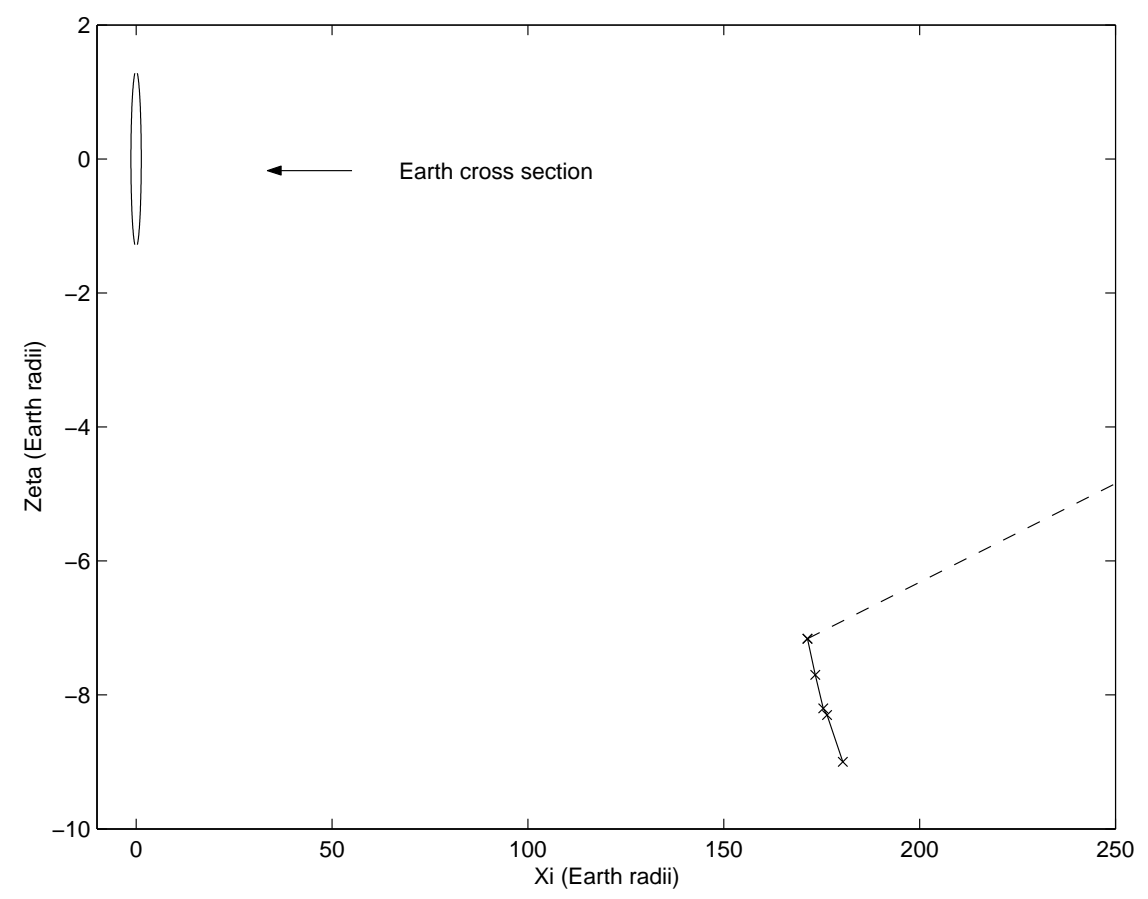

Fig. 11. For the asteroid $2003 \mathrm{UM}_{3}$, after finding the LOV point of minimum distance to the Earth on the TP of the 2034 encounter (the regula falsi iterations are marked with crosses joined by lines), the first iteration of the Newton's method to search for a VI representative fails disastrously (dashed line), going off in a direction very different from the one towards the Earth. At the second iteration Newton's method ends up not having at all an encounter (within $0.2 \mathrm{AU}$ ) in 2034. Then this VI is rated as spurious.

Case 1 has an additional complexity, in that a fold on the TP can be either towards or away from the Earth (see Figures 4 and 5). In the first case the existence of the fold does not imply that the VI is spurious, actually we expect 
two separate VIs. We use a control on the number of direction reversals, after a number of them (e.g., 7) we conclude that the Newton iterations are "bouncing" off the fold and the Earth is actually protected by the fold. However, sometimes a VI representative is found after a few reversals, as in Figure 12. Also in the case of Figure 4 it was possible to find one VI representative only after a number of reversals.

To calculate the probability of a VI found off-LOV, with the algorithm seen above, we have to apply a correction to the linearized probability integral computed as described in Section 6. The purpose is to restore the relationship between the cost function of the least squares fit and the probability density, which also applies in the nonlinear case. Specifically, the correction factor we apply is

$$
k_{o f f}=\exp \left[-\frac{1}{2}\left(\chi^{2}-\sigma^{2}-\sigma_{i m p}^{2}\right)\right]
$$

where $\chi$ is, up to a factor depending on the number of observations, the RMS of the residuals, $\sigma$ is the VI parameter on the LOV and $\sigma_{i m p}$ is the lateral distance from the LOV to the Earth impact cross section divided by the semiwidth $w$. This correction factor, however, is only a rough approximation of the full recomputation of the probability integral which would be needed for a rigorous probability estimate. Thus, in cases in which the nonlinearity is strong in the direction transversal to the LOV, this correction may not be reliable.

\subsection{Sentry Spurious VI Filtering}

Sentry examines the local behavior and topology of the LOV in an effort to verify that the linear assumptions that went into the local analysis, and resulted in a VI detection, are valid. If they are not, then the VI detection is judged unreliable and is rejected, even though the VI may in fact exist.

Under this strategy, a series of linearity tests are conducted, based on the LOV characteristics at the end points, $\sigma_{1}$ and $\sigma_{2}$, of the finally converged regula falsi interval. By the definition of the Sentry convergence criterion, these points must be within about $640 \mathrm{~km}$ (0.1 Earth radii) of each other on the Earth TP, and so estimating derivatives by taking finite differences will be reliable. Of course there is one fully sufficient condition for validation of a VI, and that is that the converged solution represents an actual impactor, in which case the VI is always published.

We define three parameters, the curvature $\mathcal{C}$, the acceleration $\mathcal{A}$ and the convergence accuracy $\theta_{\text {err }}$, and their limiting values that are used in the linearity tests as follows: 


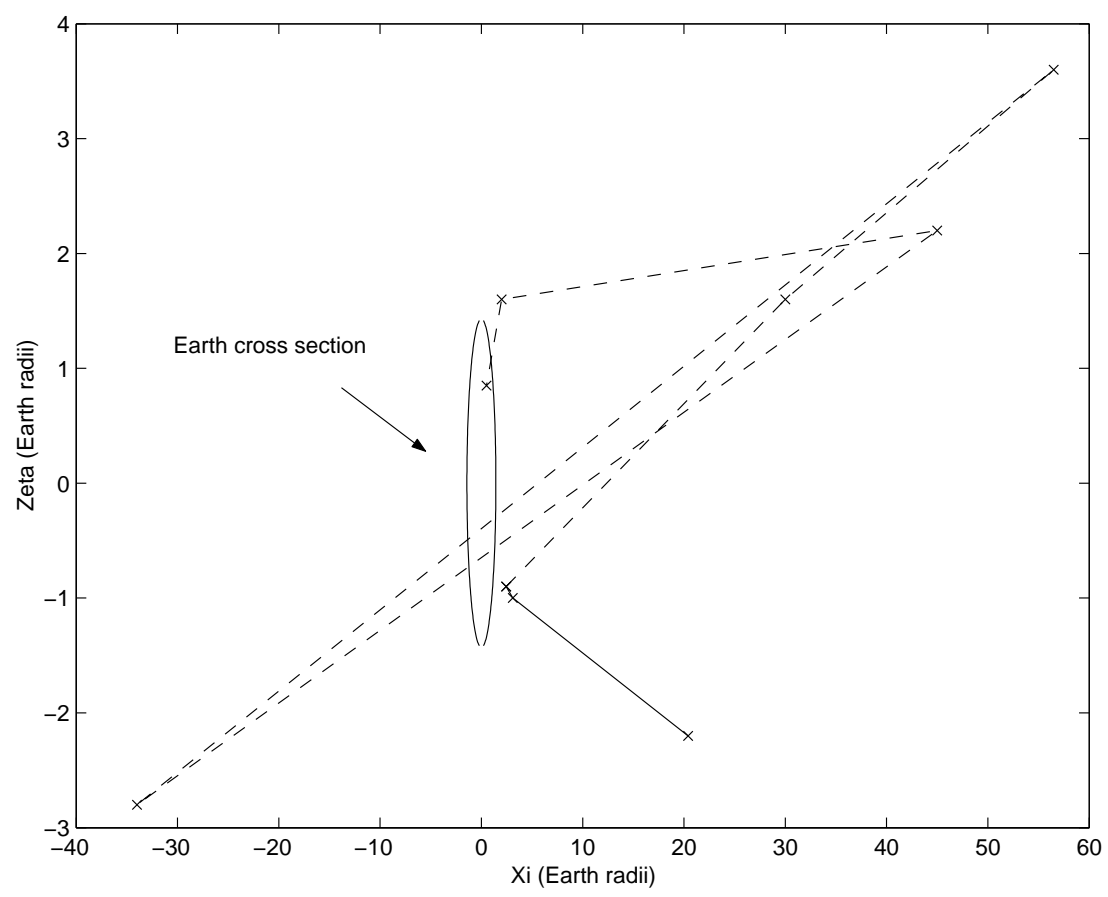

Fig. 12. For asteroid $2003 \mathrm{WY}_{153}$, after regula falsi (continuous line) finds the LOV point of minimum distance to the Earth on the TP of the 2034 encounter, Newton's method (dotted) repeatedly moves away in a direction wrong by more than $90^{\circ}$. Nevertheless, iteration 6 provides a VI representative.

$$
\begin{aligned}
\mathcal{C} & =\left|\frac{\left(\beta_{2}-\beta_{1}\right)}{\bar{\Lambda}\left(\sigma_{2}-\sigma_{1}\right)}\right|<80 \mathrm{AU}^{-1} \\
\mathcal{A} & =\left|\frac{\delta \sigma_{2}-\delta \sigma_{1}}{\sigma_{2}-\sigma_{1}}\right|<30 \\
\theta_{\text {err }} & =\left|\frac{\pi}{2}-\right| \theta||<1^{\circ} .
\end{aligned}
$$

Here $\bar{\Lambda}$ is the mean stretching of the two TP points and $\delta \sigma$ is the linearized distance along the LOV to the minimum distance point, given by $\delta \sigma=r \cos \theta / \Lambda$, where $\theta$ is the angle between $\vec{P}$ and $\vec{S}$.

For the curvature $\mathcal{C}$, the denominator is the physical distance between the TP points (which is also Sentry's regula falsi convergence criterion), and so the parameter itself is the rate at which $\beta$ changes as the LOV is traversed. If $\mathcal{C}$ is large then the direction of $\vec{S}$ is varying rapidly along the LOV. In a similar manner, the acceleration $\mathcal{A}$ measures the ratio of the actual and predicted TP separations of the two points. If $\mathcal{A}$ is far from unity, then $\vec{S}$ is varying rapidly in magnitude. The convergence accuracy $\theta_{\text {err }}$ indicates whether the LOV, as defined by $\vec{S}$, is actually orthogonal to $\vec{P}$, as would be expected. If any of the three tests above fail it is an indication of extreme nonlinearity or-more often - that the TP trails consists of unrelated points, and so the VI is rejected as spurious. 
The final filter is on the distance $d$ from the LOV to the VI. If $d>0.0086$ AU (200 Earth radii) then experience has shown that there is a substantial likelihood that a Whitney fold is present and the VI can be spurious. This is simpler and faster than actually verifying the presence of a fold, as CLOMON2 does (see Section 7.1), although there is, of course, some possibility that the putative VI may be real. However, for a VI to be detected with a large $d$ the semi-width $w$ must also be large, and since $w$ is in the denominator of the local probability density, any VI associated with such a large $w$ would have an impact probability that is considerably reduced compared to a VI with a moderate semi-width. Thus such erroneous rejections are in practice well below Sentry's generic completion level, although this is certainly not a rigorous claim.

\section{CONCLUSIONS}

Overall, we believe the second generation impact monitoring systems CLOMON2 and Sentry, whose underlying theory is described in this paper, have been a very significant improvement with respect to the first generation CLOMON. The two systems, as used operationally, have been very successful, contributing to a substantial increase in the efficiency and reliability of the process of VI detection and then elimination through additional observations. In the years 2002 and 2003, with the indispensable help of countless dedicated follow-up observers, these monitoring systems have solved more than one hundred cases of asteroids with VIs in the years 2002 and 2003 (138 for Sentry and 115 for CLOMON2). In most cases the follow up would have taken place anyway, but in a significant fraction of the cases the fact that the asteroid was on our "risk pages" did provide a strong additional motivation to the observers. In a few cases, there has been the need for a specific observation campaigns coordinated by the Spaceguard Central Node to remove the VI.

It is worth mentioning that a high level of cooperation exists among the operators of the two systems. Indeed, both teams are automatically informed by e-mail whenever new results are obtained by either system. This facilitates rapid cross-checking, enabling a prompt resolution whenever relevant discrepancies are detected. Overall, the outputs of the two systems are consistently in excellent agreement, especially for VIs representing higher levels of hazard. The statistical nature of the searches and the different techniques employed mean that, for VIs below the generic completion threshold, the list of VIs detected may be different.

The major outstanding issue, which may require a substantial amount of additional work to be solved, is to guarantee completeness of the scan for VIs down to some level of probability. We can claim that the principle of simplest 
geometry allows us to explore the most likely configurations; however, we do not have a quantitative estimate of the size (in initial conditions space) and thus of the probability of the VIs which would result from the more exotic possibilities we have not explored. Again the loss of one specific VI is not critical for the purpose of a warning system, and most of the NEA with VIs have many of them.

Another issue is the lower reliability of our impact monitoring systems whenever the confidence region is too "wide". The line of research that led to CLOMON, and later to CLOMON2 and Sentry, was started in the first half of 1998 , following the debate on the possibility of collision of $1997 \mathrm{XF}_{11}$ with the Earth after the 2028 close encounter, and was continued with the analysis of the case of $1999 \mathrm{AN}_{10}$. The basic problem presented by these cases was that of resonant and nonresonant returns (Milani et al., 1999). In fact, on the basis of the observations known at the time, it could be safely concluded that neither of these NEAs could have a "direct hit", that is, a collision with the Earth at the next close encounter with our planet (in 2028 for $1997 \mathrm{XF}_{11}$ and in 2027 for $1999 \mathrm{AN}_{10}$ ). This conclusion was based on the relatively large values of the MOID coupled with small widths of the footprints on the TP of the respective confidence regions. The small widths were, in turn, due to relatively long observed arcs. Impacts could only have happened at close encounters following the initial one, provided that the MOID in the meantime had reduced to nearly zero.

Things have not always been so simple afterwards, when impact monitoring has become a routine. For many of the newly discovered NEAs, the observed arc available when the Minor Planet Electronic Circular announcing the discovery is issued is very short. In such cases, the unidimensional sampling of the confidence region may not be suitable because the confidence region has a significant extent in two independent directions. The one dimensional sampling along some LOV can provide information on all the possible impacts only if the algorithms are able to handle the VIs lying at a significant distance transversally to the LOV. For both CLOMON2 and Sentry, when the width is large (hundreds to thousands of Earth radii) the algorithms to remove spurious VIs are not fully reliable, thus it is possible that the list of VIs found is either incomplete or contains some spurious cases.

Since the need to perform impact monitoring on asteroids observed for a very short arc is likely to persist in the near future, even to become more frequent as a result of the next generation surveys, we plan to address in our future research the development of new algorithms with better capabilities in handling off-LOV VIs. 


\section{A APPENDIX: CONVERSION FROM MTP TO TP}

The origin of MTP and TP reference frame is conventionally in the center of the Earth, but the orientation of coordinate systems on these planes is arbitrary. There are several ways to fix the reference systems (see Milani et al 2002 for more details).

To switch from MTP to TP, we use the following map (including the relationship between unperturbed velocity $U$ and closest approach velocity $V$ )

$$
(x, z, V) \mapsto(\xi, \zeta, U)
$$

expressed by

$$
\xi=\frac{b}{d} x \quad \zeta=\frac{b}{d} z \quad U=\frac{d}{b} V
$$

where $b$ is the impact parameter, $d$ is the distance of closest approach and

$$
\frac{b}{d}=\sqrt{\frac{V^{2} d}{V^{2} d-2 G m_{\oplus}}} \quad \frac{d}{b}=\sqrt{1-\frac{2 G m_{\oplus}}{V^{2} d}}
$$

The map can be explicitly written as

$$
\begin{aligned}
\xi & =\sqrt{\frac{V^{2} x^{2} \sqrt{x^{2}+z^{2}}}{V^{2} \sqrt{x^{2}+z^{2}}-2 G m_{\oplus}}} \quad \zeta=\sqrt{\frac{V^{2} z^{2} \sqrt{x^{2}+z^{2}}}{V^{2} \sqrt{x^{2}+z^{2}}-2 G m_{\oplus}}} \\
U & =\sqrt{V^{2}-\frac{2 G m_{\oplus}}{\sqrt{x^{2}+z^{2}}}}
\end{aligned}
$$

The first derivatives of the expressions above are used to transform the covariance and normal matrices for $(x, z, V)$ into the matrices for $(\xi, \zeta, U)$.

CLOMON2 works on the TP with lengths measured in Earth Radii, thus the Earth impact cross section does not have radius equal to 1 ; we have to apply a correction to take into account the gravitational focusing, so the Earth impact disk has radius

$$
b_{\oplus}=r_{\oplus} \sqrt{1+\frac{v_{e}^{2}}{U^{2}}},
$$

where $v_{e}$ is the escape velocity from the surface of the Earth:

$$
v_{e}^{2}=\frac{2 G m_{\oplus}}{r_{\oplus}} .
$$

Sentry uses a rescaling of the TP coordinates so that $b_{\oplus}$ is the unit of length on the TP, as discussed in Milani et al.(2002), thus the Earth impact disk has 
radius 1 . This difference of units has to be taken into account when comparing the risk pages of Pisa and JPL, e.g., in comparing the numerical values of stretching and width for the same VI ${ }^{14}$.

\section{B THE BEHAVIOR OF THE STRETCHING AT SUCCESSIVE ENCOUNTERS}

The derivatives of the coordinates $\xi^{\prime \prime}, \zeta^{\prime \prime}$ on the $b$-plane of the subsequent encounter, for the case $\xi_{0}^{2}>>c^{2}$, are given by (Valsecchi et al. 2003):

$$
\begin{aligned}
& \frac{\partial \xi^{\prime \prime}}{\partial \xi} \approx 1+O(c / b) \\
& \frac{\partial \xi^{\prime \prime}}{\partial \zeta} \approx O(c / b) \\
& \frac{\partial \zeta^{\prime \prime}}{\partial \xi} \approx-h s \cdot \frac{4 c \xi \zeta \sin \theta}{b^{4}}+\frac{\partial \zeta^{\prime}}{\partial \xi} \\
& \frac{\partial \zeta^{\prime \prime}}{\partial \zeta} \approx h s \cdot \frac{2 c\left(\xi^{2}-\zeta^{2}\right) \sin \theta}{b^{4}}+\frac{\partial \zeta^{\prime}}{\partial \zeta},
\end{aligned}
$$

where

$$
\begin{gathered}
s=\frac{2 \pi a^{\prime 5 / 2}\left[U \cos ^{2} \theta^{\prime}+\cos \theta^{\prime}\left(1-U^{2}\right)-3 U\right]}{\sin \theta^{\prime}} \approx 2 \pi a^{5 / 2}\left(1+\frac{10 a U c \zeta \sin \theta}{b^{2}}\right) \\
\cdot \frac{\left[U b^{2}\left(\cos ^{2} \theta-3\right)+b^{2}\left(1-U^{2}\right) \cos \theta+2 c \zeta\left(1-U^{2}+2 U \cos \theta\right) \sin \theta\right]}{b^{2} \sin \theta-2 c \zeta \cos \theta}
\end{gathered}
$$

and the auxiliary derivatives $\partial \zeta^{\prime} / \partial \xi$ and $\partial \zeta^{\prime} / \partial \zeta$ are given by

$$
\begin{aligned}
& \frac{\partial \zeta^{\prime}}{\partial \xi} \approx 2 \cos \theta \tan \phi+O(c / b) \\
& \frac{\partial \zeta^{\prime}}{\partial \zeta} \approx 1+O(c / b) .
\end{aligned}
$$

Summing up, we have that

$$
\frac{\partial \xi^{\prime \prime}}{\partial \xi} \approx 1+O(c / b)
$$

$\overline{14}$ The range of values of $b_{\oplus}$ is given in a note on the bottom of the CLOMON2 risk page. 


$$
\begin{aligned}
& \frac{\partial \xi^{\prime \prime}}{\partial \zeta} \approx O(c / b) \\
& \frac{\partial \zeta^{\prime \prime}}{\partial \xi} \approx-\frac{4 h s c \xi \zeta \sin \theta}{b^{4}}+2 \cos \theta \tan \phi+O(c / b) \\
& \frac{\partial \zeta^{\prime \prime}}{\partial \zeta} \approx \frac{2 h s c\left(\xi^{2}-\zeta^{2}\right) \sin \theta}{b^{4}}+1+O(c / b) .
\end{aligned}
$$

The determinant of the matrix of derivatives is

$$
\begin{aligned}
\frac{\partial \xi^{\prime \prime}}{\partial \xi} \frac{\partial \zeta^{\prime \prime}}{\partial \zeta}-\frac{\partial \zeta^{\prime \prime}}{\partial \xi} \frac{\partial \xi^{\prime \prime}}{\partial \zeta} \approx & {[1+O(c / b)] \cdot\left[\frac{2 h s c\left(\xi^{2}-\zeta^{2}\right) \sin \theta}{b^{4}}+1+O(c / b)\right] } \\
& +O(c / b) \cdot\left[\frac{4 h s c \xi \zeta \sin \theta}{b^{4}}+2 \cos \theta \tan \phi+O(c / b)\right]
\end{aligned}
$$

that is in general different from 1 , mostly due to the contribution coming from the diagonal terms. However, for $\xi_{0}^{2}>>c^{2}$ the two roots $\zeta_{1,2}$, those also present in the wire approximation, are located very close to $\pm \xi_{0}$, so that the term $\xi^{2}-\zeta^{2}$ vanishes, forcing the determinant to approach unity.

\section{ACKNOWLEDGMENTS}

This research was supported by the Spanish Ministerio de Ciencia y Tecnología and by the European funds FEDER through the grant AYA2001-1784. This research was conducted in part at the Jet Propulsion Laboratory, California Institute of Technology under a contract with NASA. SRC is grateful to A.B. Chamberlin, P.W. Chodas and D.K. Yeomans for their assistance in fielding the Sentry system. We thank the referees (T. Spahr and K. Muinonen) for their constructive comments.

\section{REFERENCES}

Bonanno, C. 2000. An analytical approximation for the MOID and its consequences. Astron. Astrophys. 360, 411-416.

Chamberlin, A. B., Chesley, S. R., Chodas, P. W., Giorgini, J. D., Keesey, M. S., Wimberly, R. N. and Yeomans, D. K. 2001. Sentry: An Automated Close Approach Monitoring System for Near-Earth Objects BAAS, 33, 1116.

Chesley, S.R. and Milani, A. 2000. An automatic Earth-asteroid collision monitoring system. $B A A S, 32,0602$. 
Conte, S.D. and de Boor, C. 1972. Elementary Numerical Analysis 2nd Edition, McGraw-Hill.

Gauss, C. F. 1809. Theoria motus corporum coelestium in sectionis conicis solem ambientum, Hamburg; also in Werke, siebenter band, Olms verlag, 1981.

Gronchi, G.-F. and Milani, A. 1999. Averaging on earth-crossing orbits, Celest. Mech. Dyn. Ast., 71, 109-136.

Marsden, B.G. 1999. A discourse on $1997 X F_{11}$. Journ. British Interplanetary Society, 52, 195-202.

Milani, A. 1999. The asteroid identification problem I: Recovery of lost asteroids. Icarus, 137, 269-292.

Milani, A. and Valsecchi, G.B. 1999. The asteroid identification problem II: Target plane confidence boundaries. Icarus 140, 408-423.

Milani, A., Chesley, S.R., and Valsecchi, G.B. 1999. Close approaches of asteroid $1999 A N_{10}$ : Resonant and non-resonant returns. Astron. Astrophys. 346, L65-L68.

Milani, A., Chesley, S.R., Boattini, A., and Valsecchi, G.B. 2000a. Virtual impactors: Search and destroy. Icarus 145, 12-24.

Milani, A., Chesley, S.R., and Valsecchi, G.B. 2000b. Asteroid Close Encounters with Earth: Risk Assessment. Planetary and Space Science 48, 945-954.

Milani, A., Chesley, S.R., Chodas, P.W., and Valsecchi, G.B. 2002. Asteroid Close Approaches and Impact Opportunities. in Asteroids III, W.F. Bottke Jr. et al. eds., Arizona University Press, pp. 55-69.

Milani, A., Sansaturio, M.E., Tommei, G., Arratia, O. and Chesley, S.R. 2004. Multiple solutions for asteroid orbits: computational procedure and applications, Astr. Astrophys., submitted.

Muinonen, K. 1999. Asteroid and comet encounters with the Earth: Impact hazard and collision probability. In Roy, A.E. and Steves, B.A., editors, NATO ASI Proc. 522: The Dynamics of Small Bodies in the Solar System, A Major Key to Solar System Studies, page 127. Kluwer.

Murray, C. D. and Dermott, S. F. 2000. Solar system dynamics, Cambridge Univ. Press, Cambridge.

Öpik, E.J.,1976. Interplanetary Encounters, Elsevier, New York.

Tommei, G. 2002. Algoritmi per il monitoraggio di possibili impatti asteroidali. 
Thesis, University of Pisa, Pisa, Italy

Valsecchi, G.B., Milani, A., Gronchi, G.-F., and Chesley, S.R. 2003. Resonant returns to close approaches: analytical theory, Astron. Astrophys. 408, 11791196.

Whitney, H., 1955. On singularities of mappings of euclidean spaces. I. Mappings of the plane into the plane, Annals of Mathematics, 62, 374-410. 